This is a post-print of the following article: W. G. Noehring; W. A. Curtin Acta Materialia 2017,, 14. 135-148.. The formal publication is available at http://dx.doi.org/10.1016/j.actamat.2017.02.027 (C) 2017. This manuscript version is made available under the CC-BY-NC-ND 4.0 license http://creativecommons.org/licenses/by-nc-nd/4.0/

\title{
Dislocation cross-slip in fcc solid solution alloys
}

\author{
Wolfram Georg Nöhring ${ }^{\mathrm{a}, *}$, W. A. Curtin ${ }^{\mathrm{a}}$ \\ ${ }^{a}$ Institute of Mechanical Engineering, École Polytechnique Fédérale de Lausanne, EPFL STI IGM Station 9, CH-1015 Lausanne
}

\begin{abstract}
Cross-slip is a fundamental process of screw dislocation motion and plays an important role in the evolution of work hardening and dislocation structuring in metals. Cross-slip has been widely studied in pure FCC metals but rarely in FCC solid solutions. Here, the cross-slip transition path in solid solutions is calculated using atomistic methods for three representative systems of $\mathrm{Ni}$ - $\mathrm{Al}$, $\mathrm{Cu}-\mathrm{Ni}$ and $\mathrm{Al}-\mathrm{Mg}$ over a range of solute concentrations. Studies using both true random alloys and their corresponding average-alloy counterparts allows for the independent assessment of the roles of (i) fluctuations in the spatial solute distribution in the true random alloy randomness and (ii) average alloy properties such as stacking fault energy. The results show that the solute fluctuations dominate the activation energy barrier, i.e. there are large sample-to-sample variations around the average activation barrier. The variations in activation barrier correlate linearly with the energy difference between the initial and final states. The distribution of this energy difference can be computed analytically in terms of the solute/dislocation interaction energies. Thus, the distribution of cross-slip activation energies can be accurately determined from a parameter-free analytic model. The implications of the statistical distribution of activation energies on the rate of cross-slip in real alloys are then identified.
\end{abstract}

Keywords: Screw dislocation, Dislocation mobility, Face-centered cubic crystals, Solid solution, Modeling

\section{Introduction}

Materials design for enhancing the performance of metals used in load bearing structural components requires mechanistic understanding of the underlying microscopic processes that control the mechanical properties. Plastic deformation in polycrystalline metal alloys is controlled by the motion of, and interactions between, lattice dislocations. There are many different aspects of dislocation motion and interactions. One important process is cross-slip, the process by which dislocations change glide planes. Cross-slip is restricted to dislocations having screw character, where the dislocation line direction $\xi$ is parallel to the dislocation Burgers vector $\mathbf{b}, \boldsymbol{\xi} \cdot \mathbf{b}=0$, so that the dislocation glide plane $\boldsymbol{\xi} \times \mathbf{b}$ is not uniquely defined. Cross-slip contributes to dislocation multiplication, due to double-cross-slip [1], and to dislocation annihilation, due to cross-slip of oppositely-signed screw dislocation gliding on nearby parallel planes [2]. Crossslip also allows dislocations to overcome obstacles, such as precipitates [3, 4]. These microscopic processes determine the macroscopic stress-strain evolution of the material, and this has driven the extensive study of cross-slip processes and mechanisms in metals [5-28]

In FCC metals, cross-slip has been invoked to explain several meso- and macroscopic aspects of deformation, in particular specific forms and transformations of the dislocation network. For example, the prevalence of edge dislocation dipoles in stage I of FCC single crystal deformation is explained by mutual annihilation of screw segments through cross-slip [29-31].

\footnotetext{
${ }^{*}$ Corresponding author

Email address: wol fram.nohring@epf l. ch (Wolfram Georg Nöhring )
}

Other structures whose formation is thought to involve crossslip are dislocation sheets [32] and persistent slip bands [33]. Recently, the importance of cross-slip for network formation has been shown using dislocation dynamics simulations [23] and dislocation-based plasticity models [34]. Cross-slip has also been incorporated into models for macroscopic plastic behavior of FCC metals, specifically work hardening and dynamic recovery [35-37], creep at intermediate homologous temperatures [38-41] ${ }^{1}$ and the copper-brass texture transition [45-48]. Crossslip is also postulated to be involved in the transition between stage II and stage III deformation, see e.g. [8, 49-51] and [52], p. 154, although there is some controversy here.

Here, we focus on the cross-slip mechanism and the associated activation energy $\Delta E_{\text {act }}$. Any higher-level modeling effort that strives to be predictive requires this fundamental information. Specifically, $\Delta E_{\text {act }}$ for the appropriate length of cross-slipping segment (see below) is required to calculate the rate $r$ of cross-slip. Since cross-slip is a thermally activated mechanism, this rate can be described by an Arrhenius law of the form

$$
r(T)=v \exp \left(-\frac{\Delta E_{\mathrm{act}}}{k T}\right),
$$

where $k$ is the Boltzmann constant, $T$ is temperature, and $v$ is an attempt frequency.

The most prominent mechanism for cross-slip in FCC is the Friedel-Escaig [7, 8] (F-E) mechanism. The F-E mechanism assumes that the initial dislocation, dissociated into Shockley partial dislocations on an initial glide plane [53], must constrict

\footnotetext{
${ }^{1}$ See also the discussion in $[42,43]$ as well as the review [44].
} 
This is a post-print of the following article: W. G. Noehring; W. A. Curtin Acta Materialia 2017, 14. 135-148.. The formal publication is available at http://dx.doi.org/10.1016/j.actamat.2017.02.027 (C) 2017. This manuscript version is made available under the CC-BY-NC-ND 4.0 license http://creativecommons.org/licenses/by-nc-nd/4.0/

locally to form a Stroh constriction [54] from which the dislocation can cross-slip and begin dissociating on another glide plane. The transition state of the F-E mechanism consists of a short section of dislocation dissociated on the cross-slip plane with the remainder of the dislocation residing on the original glide plane, and with two constrictions joining the two regions segments (see Fig. 1). Escaig initially calculated the activation energy for this mechanism using a linear and isotropic elastic dislocation line tension approach. Since then, several workers have proposed improvements or more elaborate calculations [55] $[17,56]$. The F-E mechanism is also seen in atomistic transition path calculations [57].

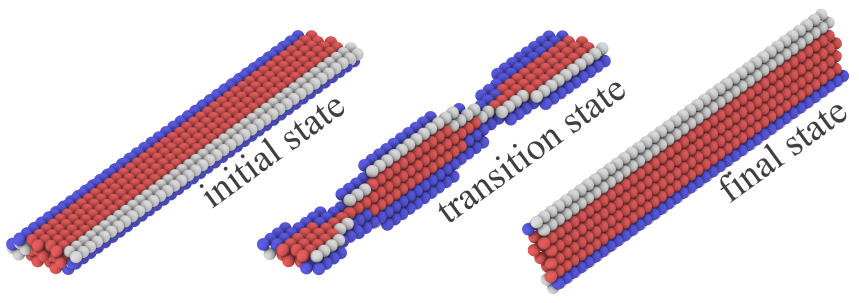

Figure 1: Typical states during dislocation cross-slip in average Ni-15 at.\% Al. The transition state was calculated with the string method [58], see Sec. 2.2. Atoms are colored according to their common neighbor value [59] (red=HCP, blue $=\mathrm{BCC}$, white $=$ other). Atoms in FCC coordination are not shown. Ovito [60] was used for visualization.

The F-E mechanism generally predicts a high activation energy, making cross-slip unlikely at room temperature. Since cross-slip is implicated in many processes at such moderate temperatures, there has been effort to examine cross-slip associated with heterogeneous mechanisms. The cross-slip energy barrier can be reduced to roughly one half of the homogeneous nucleation value if cross-slip is nucleated at a pre-existing constriction, e.g. a jog, as first considered by Escaig $[8]^{2}$. In the past two decades, atomistic models have confirmed that heterogeneities like jogs [22, 62], intersections with forest dislocations [63-66] or surfaces [67] can significantly reduce the activation energy.

In most studies to date, the metal is assumed to be pure. Extension to alloys is primarily considered by assuming that the effects of alloying are limited to changing the relevant average material properties entering the F-E model. In particular, alloying is considered mainly to change the stacking fault energy $\gamma_{\mathrm{sf}}$, with decreases in $\gamma_{\mathrm{sf}}$ leading to wider dissociation of the initial partial dislocations, and thus a higher energy for forming the constriction. A more accurate dimensionless measure for the dissociation width, and hence the tendency for cross-slip, is $\gamma_{\mathrm{s} f} / \mu b$, the ratio of stacking fault energy to shear modulus and Burgers vector magnitude [68]. However, this reduction to a single parameter is a simplistic model of the alloying effect that misses important mechanisms identified here. Surprisingly, there have been few dedicated studies of alloying effects on FCC cross-slip to date.

Andrews et al. [69] developed an elastic model to compute the energy to form a Stroh constriction using a line tension

\footnotetext{
${ }^{2}$ Note also that Escaig incorporated another kind of heterogeneity in his model by assuming that the dislocation is piled up against an unspecified obstacle on the glide plane, see [61] and the discussion therein.
}

model, but with the assumption of solute segregation to the dissociated dislocation. The assumption of segregation leads to strong pinning of the initial dislocation, leading to very high energy barriers for cross-slip (e.g. in $\mathrm{Cu}-\mathrm{Zn}$, from approximately $1.64 \mathrm{eV}$ at 0.0025 at.\% $\mathrm{Zn}$ to ca. $10.8 \mathrm{eV}$ at 20 at.\% $\mathrm{Zn}$ ). This assumption is probably not valid for considering materials being deformed steadily at normal strain rates and moderate temperatures, where solute diffusion is too slow to cause significant segregation near dislocations that are temporarily pinned at obstacles. An atomistic study of cross-slip in solid solutions without segregation was conducted by Du et al. [70], who calculated the activation energy for cross-slip in Ni-2 at.\% $\mathrm{Al}$ and Ni-10 at.\% Al, using the Nudged Elastic Band [71] method. The activation energy increased by $0.2 \mathrm{eV}$ if an Al-Al solute pair was formed in the process, due to strong Al-Al near-neighbor repulsion in $\mathrm{Ni}-\mathrm{Al}$; this result will echo our broader findings below. A similar methodology was used by Wen et al. for Ni with very ordered arrangements of interstitial $\mathrm{H}$ solutes [72, 73]. Overall, however, there are no systematic studies of cross-slip in realistic fcc solid solution alloys that would reveal the clear effects of alloying on this important process.

In the present paper, we study the effect of substitutional solutes on the cross-slip activation energy $\Delta E_{\text {act }}$ in random FCC solid solutions. We compute the activation energies in a set of model alloys (Ni-Al, $\mathrm{Cu}-\mathrm{Ni}$, and $\mathrm{Al}-\mathrm{Mg}$ ) over a range of concentrations using atomistic transition path calculations. These calculations show that the activation energy is a random variable with large fluctuations around the mean value. The large fluctuations found over the length of the critical cross-slip nucleus indicate that cross-slip in alloys can be initiated at the statistically easiest region for cross-slip along a long dislocation line. Therefore, average-alloy models are not useful for judging the ease of cross-slip in alloys, and an understanding of the distribution of activation energies is necessary. We show that the activation energy for a specific random distribution of solutes is closely correlated with the energies of the dislocation in the initial and final states. We then develop an analytic model to compute the standard deviation in energy between initial and final states, and validate the model against the simulations. The analytic model in tandem with the observed correlation then allows for an accurate estimate of the statistical variations in cross-slip activation barrier in terms only of the fundamental solute/screw-dislocation interaction energies. These energies can be obtained not only from semi-empirical interatomic potentials (as done here) but also from first-principles studies, enabling for prediction of cross-slip in alloys that do not yet exist or for which there are no reliable interatomic potentials.

The rest of the paper is organized as follows. In the first two sections, we describe the setup of the transition path calculations. The first section presents calculations using the "average atom" interatomic potential for a desired alloy, which accurately captures the cross-slip behavior in the absence of fluctuations in local solute distributions, thereby providing the average reference cross-slip barrier as if the alloy was a pure element. The second section describes the transition path calculations in the true random alloys. The resulting transition paths and activation energies are presented in Sec. 3, demonstrating the statistical 
This is a post-print of the following article: W. G. Noehring; W. A. Curtin Acta Materialia 2017,, 14. 135-148.. The formal publication is available at http://dx.doi.org/10.1016/j.actamat.2017.02.027 (C) 2017. This manuscript version is made available under the CC-BY-NC-ND 4.0 license http://creativecommons.org/licenses/by-nc-nd/4.0/

variations in activation barrier. The correlation between activation barrier and energy difference between initial and final states is also shown. An analytic model for the statistical distribution of activation energies is then derived and validated in Sec. 4. The important implications of our results are discussed in Sec. 5.

\section{Simulations of Cross-slip in FCC Alloys: Methodology}

\subsection{Selected Alloys}

Our purpose is to demonstrate the general role of alloying on cross-slip across a range of FCC alloys by using model alloy materials over a wide range of solute concentrations. Here, we consider alloys of $\mathrm{Al}+(2,6,10,14,18,22)$ at. $\% \mathrm{Mg}, \mathrm{Ni}+(2,4,8$, $10,12,15)$ at. $\% \mathrm{Al}$ and $\mathrm{Cu}+(10,22,33,68,79,90)$ at.\% $\mathrm{Ni}$ as described by the EAM potentials for $\mathrm{Al}-\mathrm{Mg}$ [74], $\mathrm{Ni}-\mathrm{Al}$ [75], and $\mathrm{Cu}-\mathrm{Ni}$ [76]. While these model materials may exceed the solute concentrations of the corresponding real alloy materials (e.g. Al$\mathrm{Mg}$ is limited to $\sim 5$ at. $\% \mathrm{Mg}$ and $\mathrm{Ni}-\mathrm{Al}$ is limited to $\sim 13$ at. $\% \mathrm{Al}$ ), the chosen systems serve to cover a range of situations that span real materials (e.g. low and high stacking fault energies; large and small solute misfit volumes; weak and strong solutesolute interaction energies). Furthermore, results will show that alloying can be significant even at low concentrations (e.g. Al2 at.\% Mg). Furthermore, the main new features of the present study are expected to be relevant to the emerging classes of fcc High Entropy Alloys (HEA) [77], which are essentially highly concentrated multicomponent solid solutions.

\subsection{Average Alloys}

We first study the cross-slip transition path in "average alloys" that are modeled using the "average-atom" (A-atom) interatomic potentials (see below) [78, 79]. These calculations serve two purposes: first, they provide the average activation energy in a homogeneous material having the same elastic constants, lattice constants, and stacking fault energies, as the true random alloy, and so differ only due to the absence of local fluctuations in the solute distribution. Second, the A-atom configurations serve as templates for building the initial and final states of the true random solutions.

In the A-atom method, the different real atom types in a true random solid solution alloy are replaced by a single equivalent "average atom". The derivation for the class of Embedded Atom Method (EAM) [80] interatomic potentials is described in [78, 79]. One assumption is made: the average embedding energy functional is linear in deviations of the electron density from the average electron density. The random alloy also implies that the solute distribution is uncorrelated, i.e. there is no segregation or short-range order. Under these conditions, the EAM functions of the A-atom are simply the concentration-weighted averages of the pure element functions. The accuracy of the A-atom method has been demonstrated for a wide range of properties [79] and at finite temperatures [81].

Transition state calculations were started as follows. Using the appropriate FCC lattice parameter $a$, a cylindrical geometry as shown in Fig. 2 was constructed. An inner core of atoms has a radius $20 b$, where $\mathrm{b}$ is the dislocation Burgers vector magnitude of a perfect dislocation in FCC. An outer shell of atoms has a thickness greater than two times the cutoff radius of the potential. Periodic boundary conditions were imposed

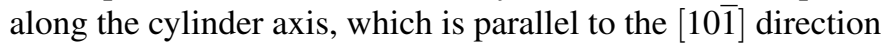
and has a length of $40 \mathrm{~b}$. This length is motivated by studying the dependence of the transition path and energy in pure $\mathrm{Ni}$. The length $40 \mathrm{~b}$ is the shortest length at which the cross-slip barrier becomes length-independent, i.e. at which there is very limited interaction between the two constrictions formed during cross-slip nucleation. For example, the energy at $40 b$ is $1.72 \mathrm{eV}$ and is only $0.02 \mathrm{eV}$ larger at $160 \mathrm{~b}$. This length is therefore a characteristic length of cross-slip nucleation denoted by $\zeta_{\mathrm{csn}}$. Different alloys may have different $\zeta_{\mathrm{csn}}$ because of their different elastic properties. For simplicity, the same length of $\zeta_{\mathrm{csn}}=40 \mathrm{~b}$ was also used for all other materials, which is ex-post-facto determined to be a good assumption.

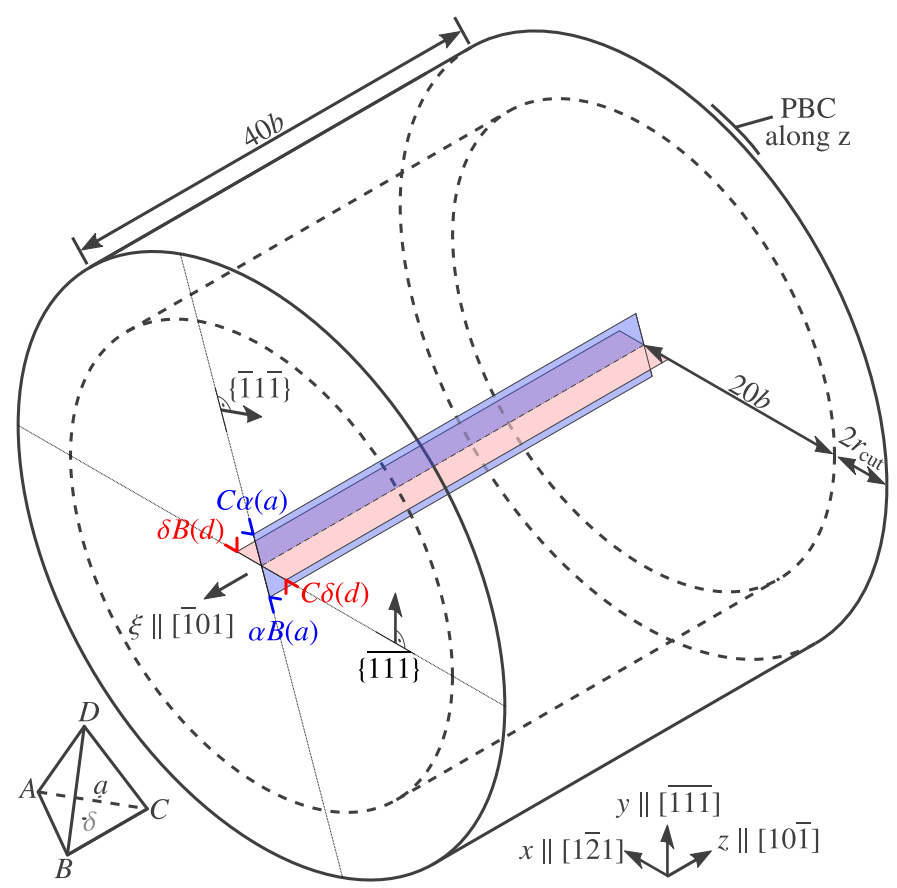

Figure 2: Sketch of the simulation cell for transition path calculations. Atoms in the outer shell of the cylinder are fixed during the calculation. In the initial state, the screw dislocation is dissociated into partial dislocations $\delta B$ and $C \delta$ on the $\{\overline{111}\}$ plane (red). In the final state, the screw dislocation is dissociated into $C \alpha$ and $\alpha B$ (blue).

A $1 / 2 a[10 \overline{1}]$ screw dislocation $(C B(a)$ or $C B(b)$ on the Thompson tetrahedron) with line direction $[10 \overline{1}]$ was then inserted into the center of the cylinder by applying the displacement field according to Stroh's [82] anisotropic-elastic solution. In the initial state, the dislocation should be dissociated on the $(\overline{111})$ glide plane and into two Shockley partial dislocations $1 / 6 a[\overline{2} 11]$ $(\delta B(d))$ and $1 / 6 a[\overline{11} 2](C \delta(d))$. In the final state, the dislocation should be dissociated on the $(\overline{1} 1 \overline{1})$ cross-slip plane and into $1 / 6 a[\overline{21} 1](C \alpha(a))$ and $1 / 6 a[\overline{1} 12](\alpha B(a))$. The dislocations were inserted as initially perfect dislocations, which preserves the physical cut plane for the initial and final states. To control dissociation during subsequent minimization, the precise geometrical center of the elastic displacement field was varied by 
This is a post-print of the following article: W. G. Noehring; W. A. Curtin Acta Materialia 2017, 14. 135-148.. The formal publication is available at http://dx.doi.org/10.1016/j.actamat.2017.02.027 (C) 2017. This manuscript version is made available under the CC-BY-NC-ND 4.0 license http://creativecommons.org/licenses/by-nc-nd/4.0/

fractions of an ångström to create dislocations dissociated on either the initial or final glide plane.

Holding the atoms in the outer shell fixed, the system energy was minimized using the conjugate gradient (CG) algorithm [83] and the FIRE algorithm [84]. Convergence was assumed when the norm of the force vector fell below $10^{-6}-10^{-8} \mathrm{eV} / \AA$. The FIRE parameters are listed in Tab. A. 1 in Appendix A. In $\mathrm{Al}-\mathrm{Mg}$ and $\mathrm{Cu}-\mathrm{Ni}$, the force norm sometimes stalled at a higher value. The convergence criterion was then increased, to $2 \cdot 10^{-4}$ $\mathrm{eV} / \AA$ in $\mathrm{Al}-\mathrm{Mg}$, and to $5 \cdot 10^{-6} \mathrm{eV} / \AA$ in $\mathrm{Cu}-\mathrm{Ni}$. All calculations were performed using LAMMPS [85].

To compute the transition path from the initial to the final state, a custom implementation (see Appendix A) of the Simplified Improved String method [58] was used. This method was chosen because the Nudged Elastic Band method [71], which is implemented in LAMMPS, was found to be numerically unstable in many cases. Briefly, the path is discretized into 32 images. Then FIRE is used to move the atoms and linear interpolation is used to enforce an equal arc-length parameterization of the transition path. Linear interpolation was also used to generate the initial guess for the path. Iterations were stopped when the two-norm of the displacement of each intermediate state relative to its position in the previous iteration was below $10^{-3} \AA$, or, if this threshold was not reached, after a total number of 300 string iterations. However, this threshold was reached in all average alloy calculations. Another measure of convergence is the two-norm of the force perpendicular to the path at the transition state, which should be zero for a perfectly converged path. In all three average alloy systems, the mean value of this norm was less than $2.3 \cdot 10^{-4} \mathrm{eV} / \AA$, and the absolute maximum was $3.2 \cdot 10^{-4} \mathrm{eV} / \AA$, in $\mathrm{Cu}-79$ at.\% $\mathrm{Ni}$.

\subsection{True Random Alloys}

Cross-slip transition path calculations with true random alloys were carried out using the A-atom initial and final states as templates. For each alloy and concentration, the A-atoms were replaced at random by real atoms at the desired alloy concentration. The atom positions of the true random alloys were then scaled by the ratio of the lattice parameter of a true random solution and the corresponding average material, since these are not exactly the same. The energy of the scaled configurations was minimized using CG and FIRE, allowing the dislocation to relax in the random solute environment. To generate a statistically representative set of results at any given concentration, 20 different random solute distributions were studied for each alloy and concentration.

Note that there remains a small error in the boundary conditions, because the dislocation displacement field was calculated using the elastic constants of the average alloy. However, the effects of this error are expected to be negligible. First, the mismatch in elastic constants is small. In Ni-Al, the maximum mismatch is $6.1 \%$ for $C_{11}$ at 15 at.\% $\mathrm{Al}$, with differences less than $1 \%$ in $C_{12}$ and $C_{44}$ at all concentrations. In $\mathrm{Al}-\mathrm{Mg}$, the maximum mismatch is $6.0 \%$ at 22 at. $\% \mathrm{Mg}$ for $C_{11}$ and $-7.5 \%$ for $C_{44}$. In $\mathrm{Cu}-\mathrm{Ni}$, the relative error is less than $1 \%$ at all concentrations. Furthermore, it will be shown below that changes of average alloy properties, and thus also errors in these properties, have only a small effect on the cross-slip activation energy.

Upon inserting the real atoms in the initial state, the dislocation can spontaneously glide from the center of the cell toward a nearby region where the particular fluctuation in the solute positions can lower the total energy of the system, even with an increase in energy due to the image interactions with the fixed boundary atoms. Since this additional energy change is not relevant for the cross-slip energy barrier, such cases were discarded. Specifically, an initial random alloy configuration was only accepted if the fully-relaxed displacement of the dislocation in the $x y$ plane was less than $2 \AA$ from the center of the cell. For $\mathrm{Cu}-\mathrm{Ni}$, such a displacement criterion was also applied to the final state. For $\mathrm{Al}-\mathrm{Mg}$ and $\mathrm{Ni}-\mathrm{Al}$, if the final state was found to be off-center then the final state energy was taken as a point further back along the transition path corresponding to a point where the dislocation was centered in the cell. In these evaluations, the displacement was calculated using the average positions of the non-FCC atoms in the dislocation core as obtained from a common neighbor analysis (CNA [59]) with cutoff $(\sqrt{0.125}+0.5) \times a$.

Finally, as with the A-atom cases, the transition path calculations were carried out using the String method as described in Appendix A. Again, iterations were stopped when the twonorm of the displacement of each image was below $10^{-3} \AA$, or after 300 string iterations. In $\mathrm{Cu}-\mathrm{Ni}, 35$ out of 120 calculations stopped at 300 iterations, in Al-Mg 36 and in Ni-Al 25. The average value of the force perpendicular to the path in the transition state was highest in Al-Mg; however, the influence of the stopping criterion was negligible. In calculations with less than 300 iterations, the average force was $7.1 \cdot 10^{-4} \mathrm{eV} / \AA$, and in those which stopped at 300 iterations, it was $8.06 \cdot 10^{-4} \mathrm{eV} / \AA$. The absolute maximum force was $3.4 \cdot 10^{-2} \mathrm{eV} / \AA$, in a calculation with $\mathrm{Ni}-15$ at.\% Al.

As in the average alloy calculations, the initial guess for the path was generated by linearly interpolating between the atomic coordinates of the initial and final state. This is the standard method, also often used in the Nudged Elastic Band method [86]. There is no proof that the converged path obtained with this initial guess is the path with the lowest possible activation energy. The problem could be aggravated in the random alloy calculations, due to the rougher potential energy landscape. However, the size scale of the transition process (as indicated by the spatial scale of the transition state) suggests that it is fluctuations on this larger scale that will be important, and these are much smoother. In the next section, we will show that the activation energy is correlated with energy difference between initial and final states, which is independent of the path. This correlation further suggests that (i) the general path is similar to that in the average alloy, and (ii) that the transition path is indeed governed by fluctuations over the scale of the transition state. Finally, some limited computations using various perturbed initial guesses lead to the same transition path. All of these features suggest that the present results are robust with respect to the choice of the initial path. 
This is a post-print of the following article: W. G. Noehring; W. A. Curtin Acta Materialia 2017, 14. 135-148.. The formal publication is available at http://dx.doi.org/10.1016/j.actamat.2017.02.027 ( ) 2017. This manuscript version is made available under the CC-BY-NC-ND 4.0 license http://creativecommons.org/licenses/by-nc-nd/4.0/

\section{Transition paths and activation energies}

\subsection{Average Alloys}

Our calculations yield two important pieces of information. First, they reveal the cross-slip mechanism to be the F-E mechanism. Fig. 1 shows the initial, transition and final state of cross-slip in Ni-15 at.\% Al. The corresponding states in the other average alloys are similar. Second, they reveal the energy barrier $\Delta E_{a c t}$, which is the maximum energy encountered along the transition path of relative energies

$$
\Delta E_{i}=E_{i}-E_{1} \quad\left(i=1 \ldots N_{S}\right),
$$

where $E_{i}$ is the potential energy of image $i$ and $N_{S}$ is the number of images. The path $\Delta E_{i}$ is usually shown as a function of the reaction coordinate

$$
\begin{aligned}
\hat{s}_{i} & =\frac{s_{i}}{s_{N_{S}}} \quad\left(i=1 \ldots N_{S}\right), \\
\text { where } \quad s_{i} & =\left\|\mathbf{x}_{i}-\mathbf{x}_{i-1}\right\|,
\end{aligned}
$$

and where $\mathbf{x}_{i}$ is the vector of atomic coordinates of image $i$ in $3 N$-dimensional space, and $\|\cdot\|$ indicates the Euclidean norm.

Fig. 3 shows representative paths for the three average alloy systems, one particular composition for each binary alloy; results for all other average-alloy systems are similar. The paths are characterized by a steep increase and decrease in energy near the start and end, respectively, and a region of small energy change near the center. The steep increase and decrease in energy correspond to constriction formation and annihilation, respectively, while the plateau in the center corresponds to moving apart of the constrictions. Note that the initial and final state have the same energy, because they are symmetrically equivalent in the homogeneous A-atom materials.

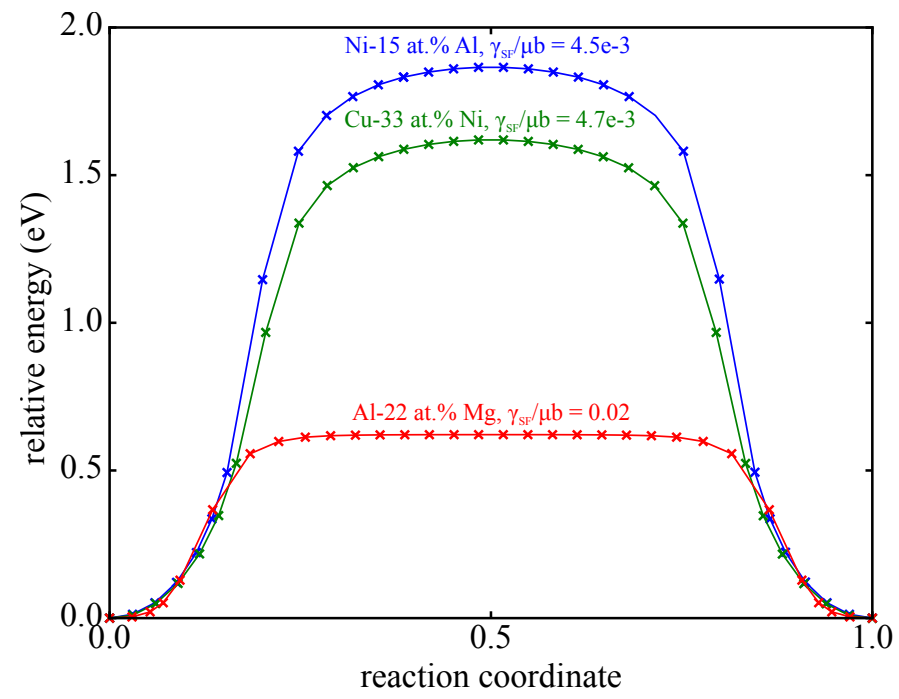

Figure 3: Relative energy along the cross-slip transition path in several average alloy systems. The reaction coordinate is the normalized displacement between the initial and final state, see Eq. 3 Note that a particular value does not correspond to the same configuration (e.g. same length on cross-slip plane) for two different paths. Thus, the shape of the paths should be compared only qualitatively.
The maximum of each curve is the activation energy $\Delta E_{\text {act }}$. As expected, it correlates with the normalized stacking fault energy $\gamma_{\mathrm{sf}} / \mu b$, which is shown in the figure. Al-22 at.\% Mg has the highest $\gamma_{\mathrm{sf}} / \mu b$ and the lowest $\Delta E_{\text {act }}$, whereas $\mathrm{Ni}-15$ at. $\% \mathrm{Al}$ has the lowest $\gamma_{\mathrm{sf}} / \mu b$ and the highest $\Delta E_{\mathrm{act}}$. The variation of $\Delta E_{\mathrm{act}}$ in each alloy system is comparatively small, see Fig. 6. For $\mathrm{Cu}-$ $\mathrm{Ni}$, the maximum change, relative to $\Delta E_{\text {act }}$ of $\mathrm{Cu}$, is $-0.08 \mathrm{eV}$, at $79 \% \mathrm{Ni}$. In Al-Mg, the maximum change is $-0.15 \mathrm{eV}$, at $18 \% \mathrm{Mg}$ and in $\mathrm{Ni}-\mathrm{Al}$, it is $0.14 \mathrm{eV}$, at $15 \% \mathrm{Al}$. As will be shown below, these changes are small compared to changes of $\Delta E_{\text {act }}$ due to compositional disorder in true random alloys.

\subsection{True Random Alloys}

Like the average-alloy calculations, the true random alloy calculations predict the F-E mechanism to be the operative crossslip mechanism. Figs. 4a) shows an example of F-E cross-slip in Ni-15 at.\% Al. Fig. 4a) and b) show the transition states from the paths with the highest and lowest $\Delta E_{\text {act }}$ in Ni-15 at.\% $\mathrm{Al}$ (3.09 and $0.86 \mathrm{eV})$. Note that the configurations are almost completely dissociated on the cross-slip and glide plane, respectively.

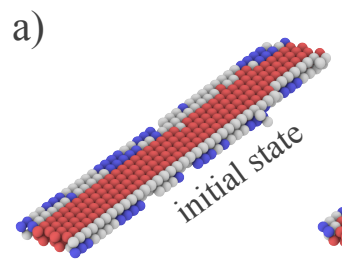

b)

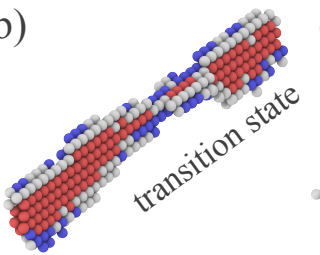

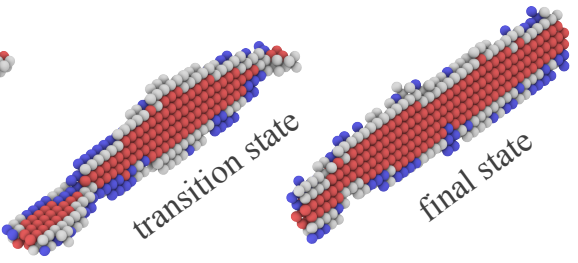

c)

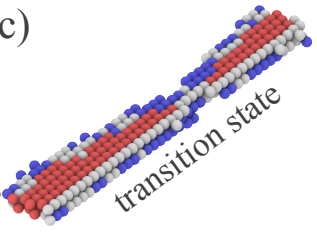

Figure 4: Typical states determined by a transition path calculation for random $\mathrm{Ni}-15$ at. $\% \mathrm{Al}$ : a) states from a path with $\Delta E_{\mathrm{act}}=1.07 \mathrm{eV}$; b) and c) transition states from the paths with the highest and lowest $\Delta E_{\text {act }}$ in Ni-15 at.\% $\mathrm{Al}$ (3.09 and $0.86 \mathrm{eV}$ ). Atoms are colored according to their common neighbor value [59] (red $=\mathrm{HCP}$, blue $=\mathrm{BCC}$, white $=$ other $).$ Atoms in $\mathrm{FCC}$ coordination are not shown . Ovito [60] was used for visualization.

The random alloy calculations predict the same F-E mechanism as found in the average alloy, but they show very different energy profiles. As an example, the transition paths of all the random samples of the Ni-15 at.\% Al alloys are shown in Fig. 5, with the final state points being corrected as described earlier. The paths are colored according to their activation energy and the transition state is marked with a circle. In contrast to the average alloy calculations, initial and final states in true random alloys have different energies, because the solute field around the dislocation is different on the glide and cross-slip plane. Most importantly, $\Delta E_{\text {act }}$ varies significantly from sample to sample.

Note that for paths with high $\Delta E_{\text {act }}$, the transition state tends to be closer to the final state. The transition states of the paths with the highest and lowest $\Delta E_{\text {act }}$ have the highest and lowest reaction coordinate, in agreement with the observation that they 
This is a post-print of the following article: W. G. Noehring; W. A. Curtin Acta Materialia 2017, 14. 135-148.. The formal publication is available at http://dx.doi.org/10.1016/j.actamat.2017.02.027 @ 2017. This manuscript version is made available under the CC-BY-NC-ND 4.0 license http://creativecommons.org/licenses/by-nc-nd/4.0/

are almost completely dissociated on the final and initial planes, respectively. This trend agrees with Hammond's [87] postulate, which states that the transition state structure is similar to the initial state when $\Delta E_{\text {act }}$ is low.

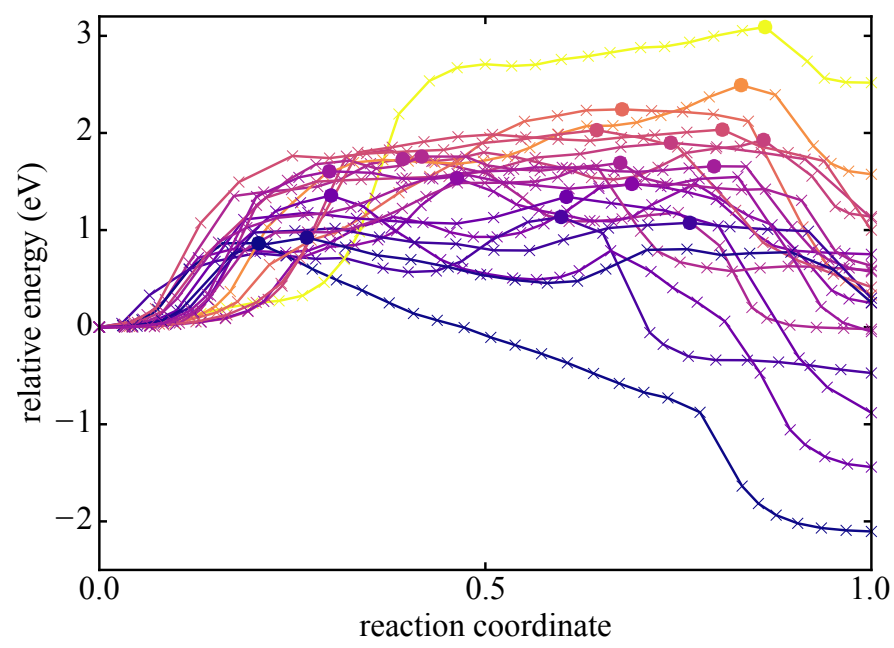

Figure 5: Transition paths for specific samples in the true random Ni-15 at.\% $\mathrm{Al}$ alloy, colored according to $\Delta E_{\text {act }}$; transition states are indicated by circles.

Fig. 6 shows $\Delta E_{\text {act }}$ as a function of concentration. In every alloy system and at every concentration, the distribution of the random alloy energies is much larger than the change of the average alloy energy relative to that of the pure metal. Even in the dilute limit, the lowest value of $\Delta E_{\text {act }}$ of the random alloy is typically several tenths of an electron volt lower than $\Delta E_{\text {act }}$ of the corresponding average alloy. The significant statistical distribution of $\Delta E_{\text {act }}$ is the most important basic result of the random alloy calculations. In the following sections, we will discuss its implications and develop a model for predicting typical values of $\Delta E_{\text {act }}$.

Finally, we note that random $\mathrm{Al}-\mathrm{Mg}$ exhibits two pecularities. In 25 out of 120 calculations, cross-slip nucleated at two sites, and these two nuclei later coalesced to form the fully-crossslipped segment. These cases are marked green in Fig. 6c). Furthermore, 17 samples have an intermediate minimum that corresponds to a partially cross-slipped state and has a negative relative energy. 5 of those samples also form two nuclei. The deepest minimum has a relative energy of $-0.39 \mathrm{eV}$. These cases are marked blue in Fig. 6c). Note that the barrier for spreading of the cross-slipped segment is then higher and more important than the barrier taken from the initial state. However, for consistency with the other results we draw the latter.
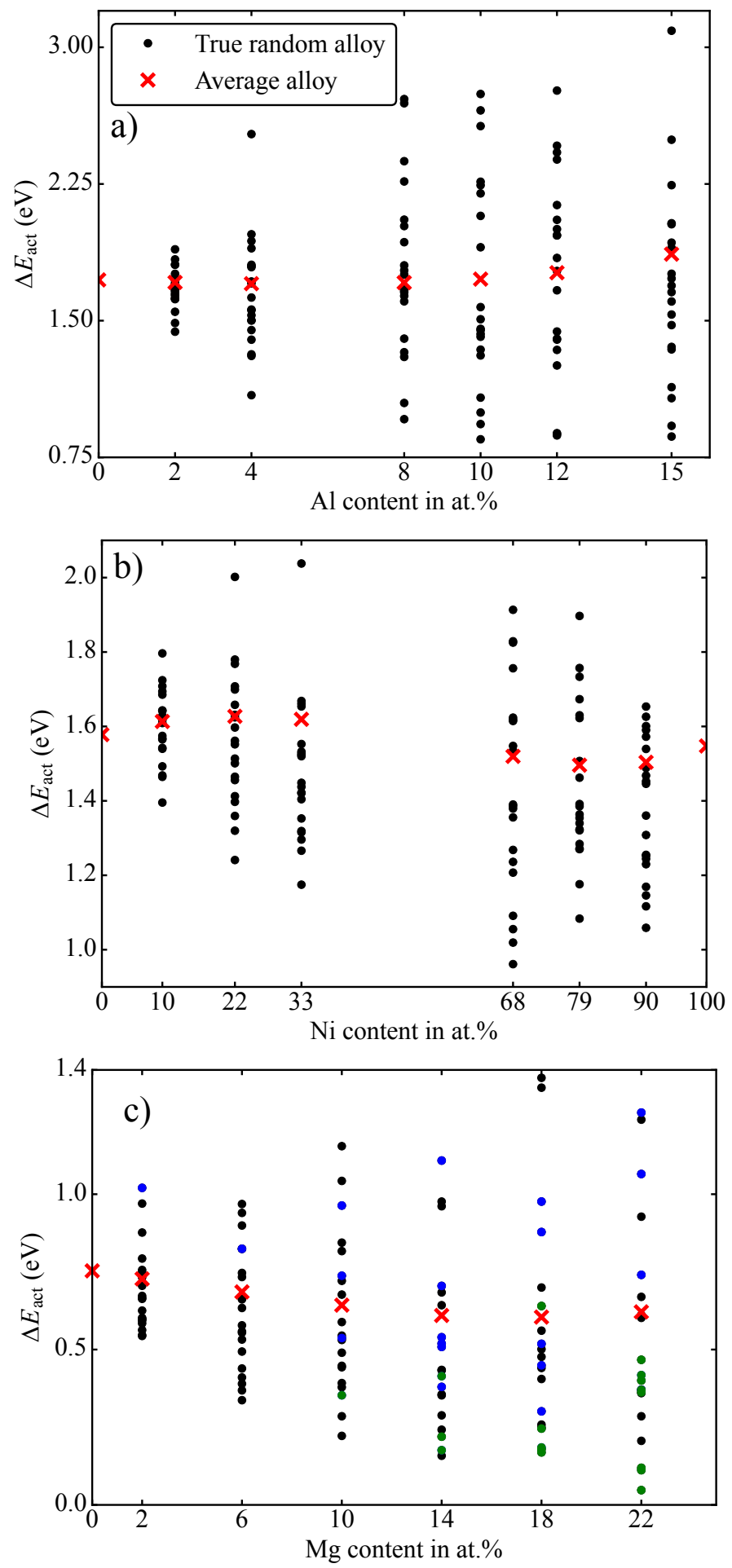

Figure 6: Cross-slip activation energies of the 20 individual samples of random alloys for (a) $\mathrm{Ni}-\mathrm{Al}$, (b) $\mathrm{Cu}-\mathrm{Ni}$, and (c) $\mathrm{Al}-\mathrm{Mg}$; also shown are the average alloy values at each composition; in (c) blue markers indicate samples with two nuclei; green markers indicate samples with a partially cross-slipped state with negative relative energy

\subsection{Preliminary Discussion}

The most striking feature of the random alloys is the large scatter of $\Delta E_{\text {act }}$ around the average value. This scatter is caused by fluctuations in the solute concentration, which are excluded 
This is a post-print of the following article: W. G. Noehring; W. A. Curtin Acta Materialia 2017, 14. 135-148.. The formal publication is available at http://dx.doi.org/10.1016/j.actamat.2017.02.027 (C) 2017. This manuscript version is made available under the CC-BY-NC-ND 4.0 license http://creativecommons.org/licenses/by-nc-nd/4.0/

a priori in the average alloys and in any models that consider only average material properties. As a consequence, $\Delta E_{\text {act }}$ in real random alloys may be significantly lower than the average

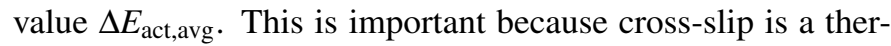
mally activated process (see Eq. 1), so that small decreases in $\Delta E_{\text {act }}$ lead to exponentially faster cross-slip rates. Along a long dislocation line, regions where the concentration fluctuations give low values of $\Delta E_{\text {act }}$ will thus dominate the rate of cross-slip nucleation. The fluctuations occur around the average $\Delta E_{\text {act }}$, so the average $\Delta E_{\text {act }}$ sets an initial scale, but the large fluctuations lead to significant lowering of $\Delta E_{\text {act }}$ in many cases, so that cross-slip nucleation is controlled by concentration fluctuations.

Accordingly, cross-slip in random alloys must be treated as a statistical problem and the effects of solute fluctuations must be accounted for. A simple model for typical values of $\Delta E_{\text {act }}$ in random alloys will be derived in the following section. Here, we continue with analysis of the simulation data. The activation energy $\Delta E_{\mathrm{act}}$ and the difference in initial and final energies, $\Delta E_{\text {end }}$, are strongly correlated, as shown in Fig. 7. If $\Delta E_{\text {end }}$ is low (more negative), then $\Delta E_{\text {act }}$ is smaller. Across all concentrations for a given alloy system, the correlation is accurately captured by a linear function,

$$
\Delta E_{\mathrm{act}}=\alpha \Delta E_{\mathrm{end}}+\Delta E_{\mathrm{ref}},
$$

where $\Delta E_{\text {ref }}$ is the activation energy at $\Delta E_{\text {end }}=0$ and $\alpha \approx 0.5$ is a constant. The value of $\Delta E_{\text {ref }}$ is just slightly lower than the average $\Delta E_{\text {act }}$ of the corresponding average alloys shown in Fig. 6; the variations of the average alloy $\Delta E_{\text {act }}$ with concentration are small so that aggregating the data across all concentrations is acceptable. A linear correlation between $\Delta E_{\text {act }}$ and $\Delta E_{\text {end }}$ is sometimes observed for chemical reactions, where it is referred to as the Bell-Evans-Polanyi [88, 89] (BEP) principle, see also [90]. The BEP principle is usually applied for simple chemical reactions of the type $A B+C \rightarrow A C+B$, and the slope $\alpha=0.5$ emerges for simple triangular transition paths.

Considering the correlation shown in Fig. 7, we can express $\Delta E_{\text {act }}$ in terms of $\Delta E_{\text {end }}$ by taking $\Delta E_{\text {ref }}=\Delta E_{\text {act,avg }}$ so that

$$
\Delta E_{\text {act }}=\Delta E_{\text {act,avg }}+0.5 \Delta E_{\text {end }} .
$$

Since the initial and final state energies are controlled by random solute concentration fluctuations, as in solute strengthening of random alloys, particular values are random variables chosen from a normal distribution [91]. The quantity $\Delta E_{\text {end }}$ is then also a random variable with standard deviation $\sigma\left[\Delta E_{\text {end }}\right]$. Assuming that $\Delta E_{\text {end }}$ follows a normal distribution, we can write the probability distribution of $\Delta E_{\text {act }}$ as

$$
P\left[\Delta E_{\mathrm{act}}\right]=\frac{1}{\sqrt{\frac{1}{2} \pi \sigma\left[\Delta E_{\mathrm{end}}\right]^{2}}} \exp \left(-\frac{\left(\Delta E_{\mathrm{act}}-\Delta E_{\mathrm{act}, \mathrm{avg}}\right)^{2}}{\frac{1}{2} \sigma\left[\Delta E_{\mathrm{end}}\right]^{2}}\right) .
$$

The statistical distribution of $\Delta E_{\text {act }}$ is thus controlled by the distribution of $\Delta E_{\text {end. }}$ In the next section, we show that $\Delta E_{\text {end }}$ can be calculated analytically, thus leading to an analytic description for the statistical distribution of $\Delta E_{\text {act }}$. The statistical distribution for $\Delta E_{\text {act }}$, which are the activation energies for initial crossslip nuclei, can then be used to determine overall distributions
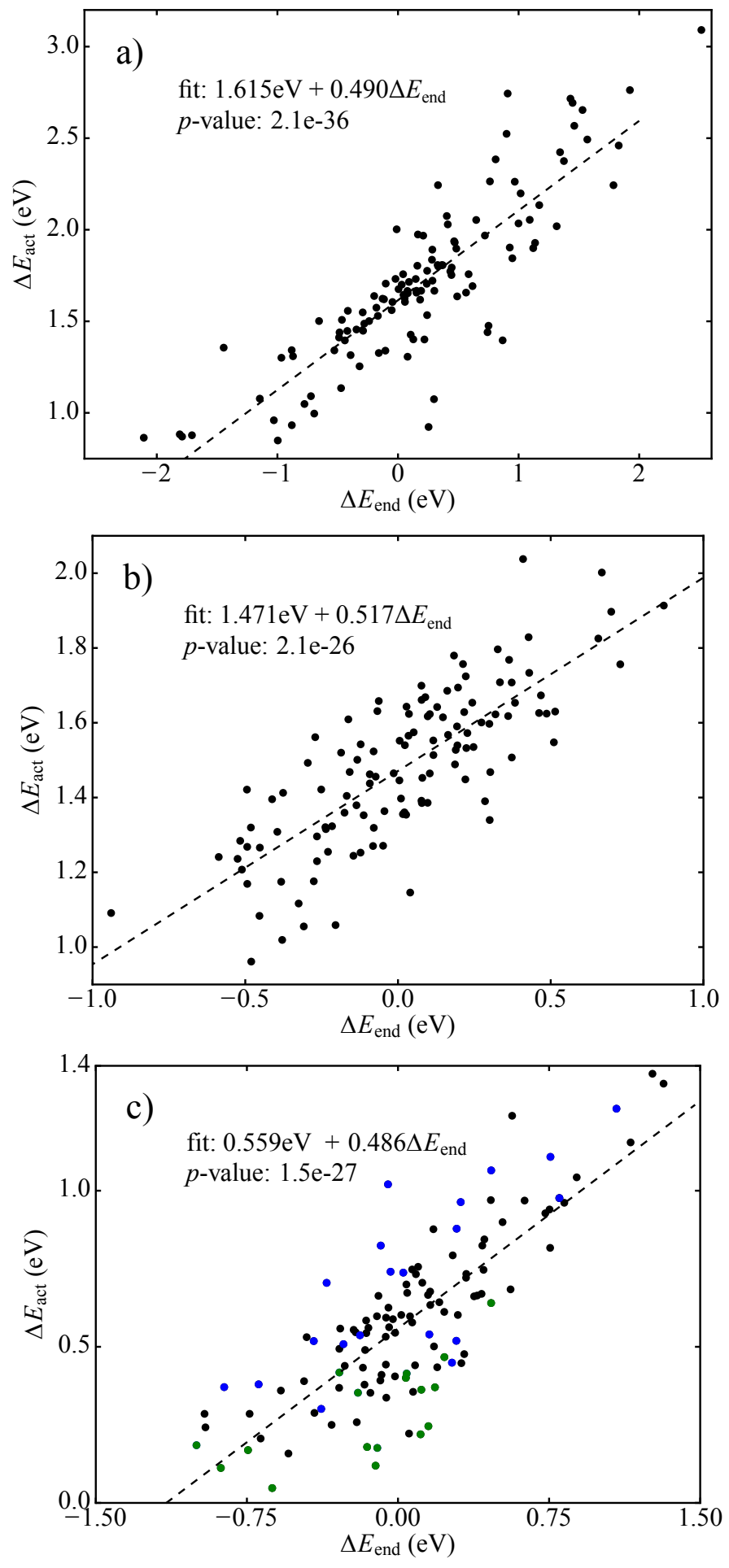

Figure 7: Cross-slip activation energy $\Delta E_{\text {act }}$ vs end state energy difference $\Delta E_{\text {end }}$, showing a linear correlation, for (a) Ni-Al, (b) $\mathrm{Cu}-\mathrm{Ni}$, and (c) $\mathrm{Al}-\mathrm{Mg}$; in (c) blue markers indicate samples with two nuclei; green markers indicate samples with a partially cross-slipped state with negative relative energy

of $\Delta E_{\text {act }}$ and the associated rate of cross-slip as a function of dislocation segment lengths, which can then be implemented into higher-level discrete-dislocation simulations to capture the effects of alloying on cross-slip. 
This is a post-print of the following article: W. G. Noehring; W. A. Curtin Acta Materialia 2017,, 14. 135-148.. The formal publication is available at http://dx.doi.org/10.1016/j.actamat.2017.02.027 (C) 2017. This manuscript version is made available under the CC-BY-NC-ND 4.0 license http://creativecommons.org/licenses/by-nc-nd/4.0/

\section{Analytic Model for the Statistical Distribution of $\Delta E_{\text {act }}$}

\subsection{General model}

Due to the correlation between $\Delta E_{\text {act }}$ and $\Delta E_{\text {end }}$, as shown in Fig. 7 and discussed in the previous section, the determination of the distribution of $\Delta E_{\text {act }}$ of the random alloy can be reduced to the problem of determining the distribution of $\Delta E_{\text {end }}$. Recall that $\Delta E_{\text {end }}$ is the difference in total energies between the final and initial states of the cross-slip. We will characterize the distribution of $\Delta E_{\text {end }}$ in terms of its standard deviation.

The end states differ in energy because, in a given distribution of random solutes, the solute-dislocation and the solutesolute interaction energies are different for the two dislocation configurations (initial and final). Since the correlation shows, $\Delta E_{\text {act }} \approx \Delta E_{\text {act,avg }}$ when $\Delta E_{\text {end }} \approx 0$, we are concerned with determination of the standard deviation of $\Delta E_{\text {end }}$ in a given alloy system at a given alloy concentration. The analysis proceeds by considering a fixed specific random distribution of solutes, as indicated in Fig. 8, computing the energy difference of the initial and final states for this particular random distribution, and then performing an analytical statistical averaging over all possible random distributions to obtain the standard deviation of $\Delta E_{\text {end }}$. There are two separate contributions to $\Delta E_{\text {end }}$ : (i) a contribution from changes in the solute/dislocation interaction energy and (ii) a contribution from changes in solute/solute interaction energy due to the fact that the change in position of the stacking fault alters the relative positions of solutes with respect to each other, especially those that are immediately on either side of the initial or final stacking fault plane. These two contributions in energy lead to two independent contributions to the standard deviation of $\Delta E_{\text {end }}$, which we denote as $\sigma\left[\Delta E_{\text {end,s-d }}\right]$ and $\sigma\left[\Delta E_{\text {end,s-s }}\right]$. The total standard deviation for $\Delta E_{\text {end }}$ is then computed as

$$
\sigma\left[\Delta E_{\text {end }}\right]^{2}=\sigma\left[\Delta E_{\text {end,s-d }}\right]^{2}+\sigma\left[\Delta E_{\text {end,s-s }}\right]^{2} .
$$

\subsection{Solute-dislocation and Solute-solute Contributions}

The contribution $\sigma\left[\Delta E_{\text {end,s-d }}\right]$ to the standard deviation of $\Delta E_{\text {end }}$ due to solute-dislocation binding energies can be modeled by adapting the solute strengthening model of Leyson and Curtin [91, 92]. Consider a slice of material parallel to the dislocation line and having a thickness of one period along the line direction, as shown in Fig. 8a). We label the atomic sites by their $\{x, y\}$ positions in the plane of the figure as $\left\{x_{i}, y_{j}\right\}$, with the origin at the center of the dislocation structure, and $x$ the direction along the glide plane. Similarly, let $z$ be the direction along the dislocation line and label the sites by their $z$ position as $z_{k}$. Define an equivalent coordinate system with respect to the cross-slipped dislocation, with positions denoted by $\left(x_{i}^{\prime}, y_{j}^{\prime}, z_{k}^{\prime}\right)$. When the dislocation cross-slips, the relative position of the atom at $\left(x_{i}, y_{j}, z_{k}\right)$ changes to $\left(x_{i}^{\prime}, y_{j}^{\prime}, z_{k}^{\prime}\right)$. If we denote the solute/dislocation binding energy as $U\left(x_{i}, y_{j}, z_{k}\right)$, then the change in total solute/dislocation binding energy is the sum over all sites given by [92]

$$
\Delta E_{\mathrm{end}, \mathrm{s}-\mathrm{d}}=\sum_{i j k} s_{i j k}\left[U\left(x_{i}^{\prime}, y_{j}^{\prime}, z_{k}^{\prime}\right)-U\left(x_{i}, y_{j}, z_{k}\right)\right]
$$
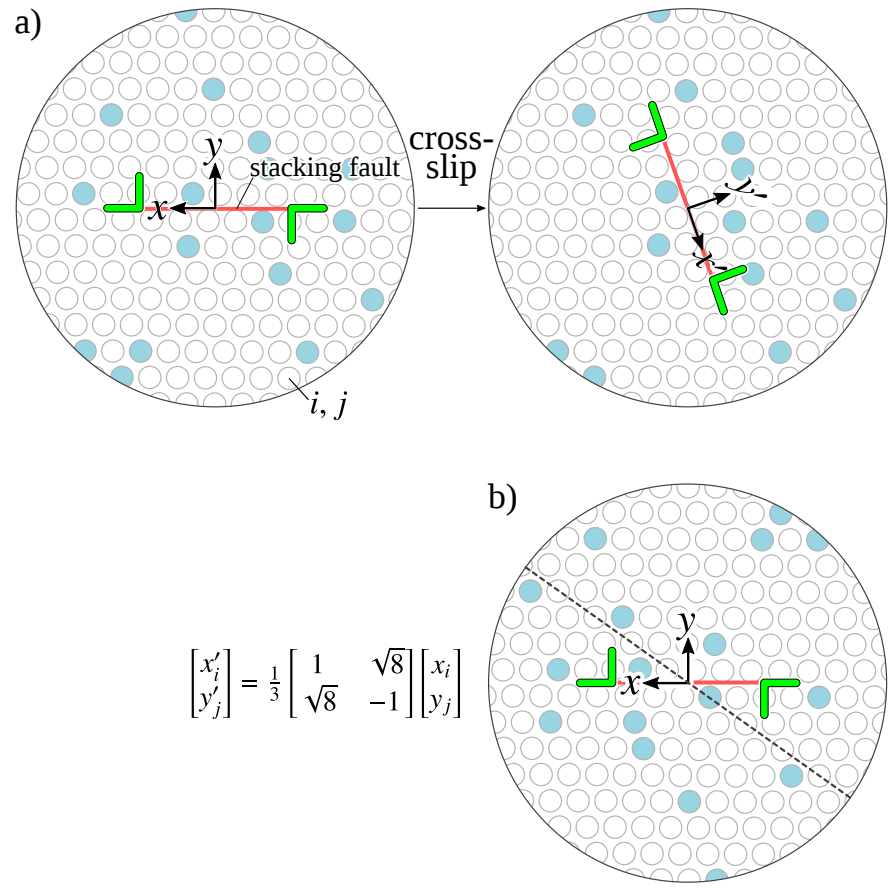

Figure 8: a) Reference frames for measuring the relative solute/dislocation positions before and after cross-slip; note that the solute distribution across sites $(i, j)$ does not change. b) The result of cross-slip is the same as a mirroring of the dislocation at $(\overline{10} \overline{1})$ (dashed line), leading to simplifications in the analysis.

where $s_{i j k}$ is the occupation variable with $s_{i j k}=1$ if a solute exists at site $(i, j, k)$ and $s_{i j k}=0$ otherwise.

If the solute concentration is high, the corresponding average alloy should be used as matrix material, so that the solutedislocation binding energies are the binding energies in an average solute environment, and with respect to the dislocation structure in that environment.

Eq. 9 gives the total energy change for a unit dislocation segment. We are interested in typical fluctuations for a dislocation with finite length $\zeta_{\mathrm{csn}}$, which corresponds to $N_{\zeta}=\zeta_{\mathrm{csn}} / b$ slices along $z$. For this line length, there are $N_{\zeta}$ sites with the same in-plane coordinate $(x, y)$, and solutes occupying any of these sites have the same solute/dislocation interaction energy. The typical energy change resulting from cross-slip of this length of dislocation is the standard deviation given by

$$
\sigma\left[\Delta E_{\text {end,s-d }}\left(\zeta_{\mathrm{csn}}\right)\right]=\sqrt{\left\langle\Delta E_{\text {end,s-d }}\left(\zeta_{\mathrm{csn}}\right)^{2}\right\rangle-\left\langle\Delta E_{\text {end,s-d }}\left(\zeta_{\mathrm{csn}}\right)\right\rangle^{2}}
$$

where $\langle\cdot\rangle$ denotes an average over all values of the occupation variables. The derivation of $\sigma\left[\Delta E_{\text {end,s-d }}\left(\zeta_{\mathrm{csn}}\right)\right]$ is analogous to the derivation of the standard deviation of the energy change during regular slip in a random alloy, see [91]. The following assumptions are made (i) the $s_{i j k}$ are uncorrelated Bernoulli random variables, (ii) $U\left(x_{i}, y_{j}, z_{k}\right)$ depends only on the atom at site $(i, j, k)$, and, as noted above, (iii) $U\left(x_{i}, y_{j}, z_{k}\right)$ is only a function of $x_{i}$ and $y_{j}$, i.e. $U\left(x_{i}, y_{j}, z_{k}\right) \rightarrow U\left(x_{i}, y_{j}\right)$ due to periodicity along $z$. We do not repeat the statistical analysis here, which involves 
This is a post-print of the following article: W. G. Noehring; W. A. Curtin Acta Materialia 2017,, 14. 135-148.. The formal publication is available at http://dx.doi.org/10.1016/j.actamat.2017.02.027 (C) 2017. This manuscript version is made available under the CC-BY-NC-ND 4.0 license http://creativecommons.org/licenses/by-nc-nd/4.0/

standard but extensive manipulations. The final result is

$$
\sigma\left[\Delta E_{\text {end,s-d }}\right]=\sqrt{c(1-c) \frac{\zeta_{\mathrm{csn}}}{b} \sum_{i j}\left[U\left(x_{i}^{\prime}, y_{j}^{\prime}\right)-U\left(x_{i}, y_{j}\right)\right]^{2}} .
$$

Note that, as shown schematically in Fig. 8, cross-slip is geometrically equivalent to performing a mirror operation of the random solute distribution across the $(\overline{1} 0 \overline{1})$ plane (see Fig. $8 \mathrm{c}$ ). This is described by the affine transformation

$$
\left[\begin{array}{l}
x_{i}^{\prime} \\
y_{j}^{\prime}
\end{array}\right]=\frac{1}{3}\left[\begin{array}{cc}
1 & \sqrt{8} \\
\sqrt{8} & -1
\end{array}\right]\left[\begin{array}{l}
x_{i} \\
y_{j}
\end{array}\right] .
$$

Due to this symmetry, the only inputs to the theory are the binding energies $U\left(x_{i}, y_{j}\right)$ in the initial state since the binding energy $U\left(x_{i}^{\prime}, y_{j}^{\prime}\right)$ at site $(i, j)$ is the binding energy at the symmetryequivalent site in the undeformed lattice. The number of required calculations can be further reduced by exploiting the fact that the configuration has two-fold rotation symmetry about a line parallel to the $z$ axis through the center. Thus, binding energies need only be calculated for the atoms in the upper half of the $x y$-plane.

The second contribution to $\Delta E_{\text {end }}$ comes from changes in direct solute-solute binding energies. For example, solute pairs (first, second,... neighbors) may be formed or destroyed along the stacking fault ribbon when it is annihilated on the glide plane and/or reformed on the cross-slip plane. Du et al. [70] have shown that this effect is important for cross-slip in Ni-Al, and Rodary et al. [93] have shown that it is important for the related problem of solute strengthening, due to the strong repulsion of Al-Al near-neighbor pairs.

Here, we restrict our attention to nearest-neighbor pairs. The contribution of pair formation and annihilation to $\Delta E_{\text {end }}$ is

$$
\Delta E_{\mathrm{end}, \mathrm{s}-\mathrm{s}}=E_{\mathrm{s}-\mathrm{s}} \Delta N_{\mathrm{s}-\mathrm{s}},
$$

where $E_{\mathrm{S}-\mathrm{s}}$ is the solute-solute binding energy and $\Delta N_{\mathrm{s}-\mathrm{s}}$ is the dimensionless net change in the number of solute pairs due to cross-slip. The contribution to $\sigma\left[\Delta E_{\text {end }}\right]$ can then be estimated as

$$
\sigma\left[\Delta E_{\text {end,s-s }}\right]=E_{\mathrm{s}-\mathrm{s}} \sigma\left[\Delta N_{\mathrm{s}-\mathrm{s}}\right],
$$

$\sigma\left[\Delta N_{\mathrm{s}-\mathrm{s}}\right]$ can be approximated using a simple model of solute pair formation and annihilation in the stacking fault of a straight dislocation, as derived in Appendix B. For an average stacking fault width $d$, we find

$$
\sigma\left[\Delta E_{\mathrm{end}, \mathrm{s}-\mathrm{s}}\right]=E_{\mathrm{s}-\mathrm{s}} \sqrt{8 c^{2}(1-c) \frac{\zeta_{\mathrm{csn} d}}{b^{2} \sqrt{3}}} .
$$

\subsection{Results and Comparison to Simulations}

The only material parameters in the model for the standard deviation of $\Delta E_{\text {end }}$ are the solute/dislocation interaction energies $U\left(x_{i}, y_{j}\right)$, the average stacking fault width $d$ and the solute pair interaction $\Delta E_{\text {end,s-s. }}$. These quantities can be obtained with atomistic or ab-initio methods; a number of examples of solute/dislocation interaction energies computed by ab initio methods [91, 92, 94, 95]. The average stacking fault width $d$ is determined using the Dislocation Extraction Algorithm [96] as implemented in Ovito [60] by taking the average distance between the extracted partial core coordinates.

For the alloys tested here, we can compute $U\left(x_{i}, y_{j}\right), d$ and $\Delta E_{\text {end,s-s }}$ as follows. The solute-dislocation binding energies were calculated starting with a cylindrical configuration as shown in Fig. 2 but with a radius of $40 b$ and a length of only $6 b$ because only individual solutes are studied and so the cell need only be slightly longer than the cut-off radius of the interatomic potential. The screw dislocation is inserted on the initial plane and relaxed, as described in Sec. 2.2. One row of atoms in the core of each partial dislocation is then fixed to prevent the dislocation from slipping towards or away from a given solute during subsequent calculations. These two rows were symmetryequivalent under two-fold rotation about the $z$-axis through the center of the dislocation. For each of the 5330 unique atomic sites in this geometry, the solute-dislocation binding is computed by inserting the desired solute atom into the desired site (removing the A-atom occupying the site), followed by fully minimizing of the energy using $\mathrm{CG}$ and FIRE until the norm of the force vector fell below $1 \cdot 10^{-6} \mathrm{eV} / \AA$, or after 200,000 iterations, and taking the difference between the potential energy after and before insertion of the solute minus the solute binding energy in the perfect A-atom FCC lattice.

Solute-solute binding energies were computed using a cube of A-atom material aligned along $\langle 100\rangle$ with edge lengths $40 a$ and fully periodic boundary conditions. A near-neighbor pair of A-atoms were then replaced by the desired real solute pair and the energy was minimized with $\mathrm{CG}$ and FIRE, stopped if the norm of the force vector fell below $1 \cdot 10^{-8} \mathrm{eV} / \AA$, or after 20,000 or 30,000 iterations. The solute-solute binding energy is then the potential energy of system containing the solute pair minus twice the energy of a single solute in the perfect A-atom FCC lattice. Recall that our explicit cross-slip transition state computations were performed on only 20 samples. To obtain accurate estimates for the statistical distribution (standard deviation) of the end state differences $\Delta E_{\text {end }}$, we have generated 100 sets of initial and final states for each alloy composition. In these cases, we impose the constraint on off-center displacements (see Sec. 2.3) for both initial and final states. In the case of $\mathrm{Cu}-\mathrm{Ni}$, the 20 sets of data from the transition path calculations were added, because there the constraint was active in both states, and thus this data was equivalent to the newly generated data.

The predicted and measured values for $\sigma\left[\Delta E_{\text {end }}\right]$ are compared in Fig. 9, and the two individual contributions from solutedislocation and solute-solute interactions are also shown. For $\mathrm{Ni}-\mathrm{Al}$, the prediction is satisfactory, with a maximum error of $0.1 \mathrm{eV}$ at 10 at.\% Al. For Ni-Al, the solute-solute interaction dominates the distribution. For Al-Mg, the prediction is also satisfactory up to 10 at. $\% \mathrm{Mg}$, with the solute/dislocation interaction dominating. At higher concentrations, the predicted standard deviation saturates while the measured value continues to increase. This deviation is due to the increasing importance of non-near-neighbor solute-solute interactions which, though individually small, are very numerous and so contribute non-negligibly to the standard deviation at higher solute con- 
This is a post-print of the following article: W. G. Noehring; W. A. Curtin Acta Materialia 2017, 14. 135-148.. The formal publication is available at http://dx.doi.org/10.1016/j.actamat.2017.02.027 ( ) 2017. This manuscript version is made available under the CC-BY-NC-ND 4.0 license http://creativecommons.org/licenses/by-nc-nd/4.0/
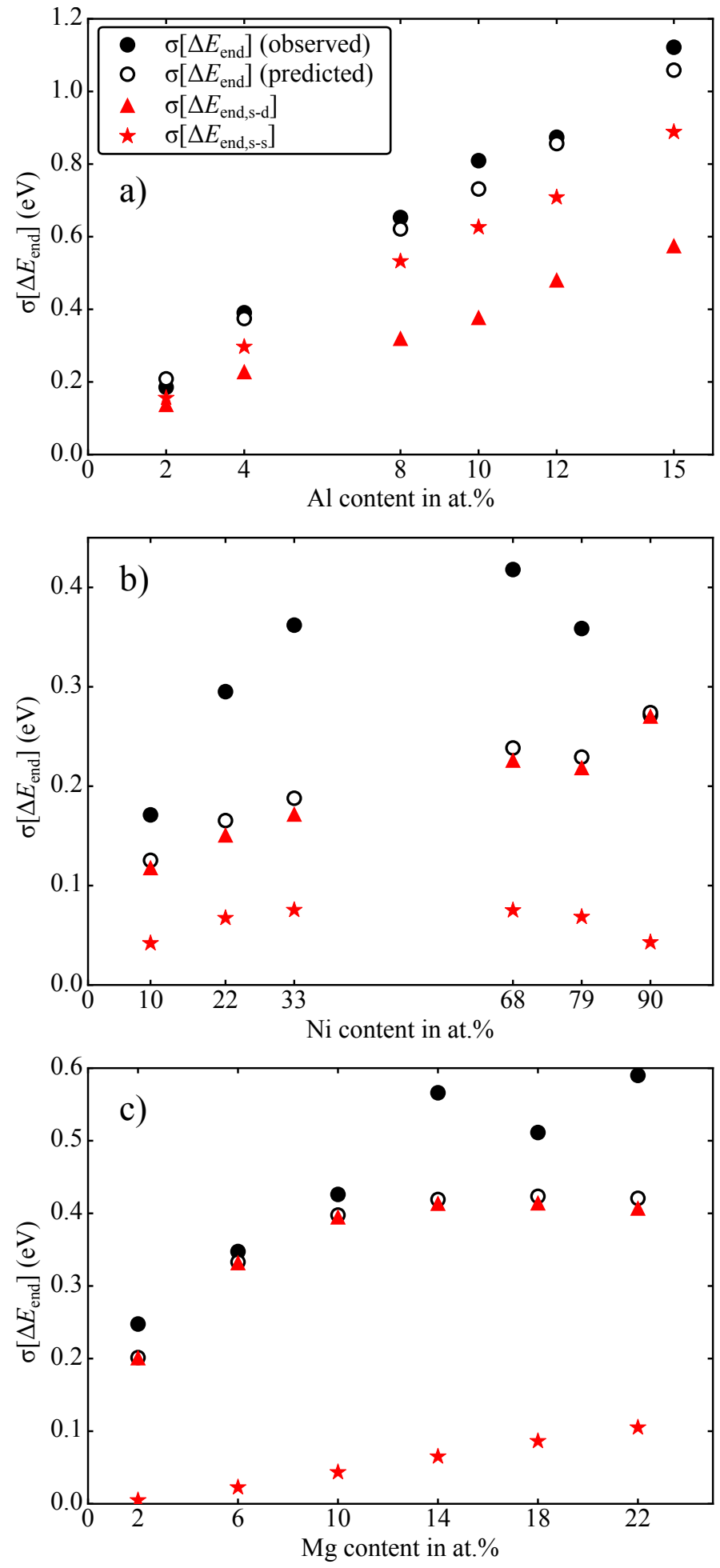

Figure 9: Observed (filled symbols) and predicted (open symbols) standard deviation of the end state energy difference $\Delta E_{\text {end }}$ in (a) $\mathrm{Ni}-\mathrm{Al}$, (b) $\mathrm{Cu}-\mathrm{Ni}$, and (c) Al-Mg. The individual contributions to the overall standard deviation, due to solute-dislocation (star symbols) and solute-solute interactions (triangle symbols) are also shown.

centrations. These interactions can be included in the analysis as a generalization of the near-neighbor pair analysis, but it is cumbersome and so not presented here. Finally, in $\mathrm{Cu}-\mathrm{Ni}$, the prediction is accurate at the lowest $\mathrm{Cu}$ and $\mathrm{Ni}$ concentrations but then deviates more significantly at intermediate concentrations. The reason for these deviations is that the solute/dislocation and solute/solute interaction energies in $\mathrm{Cu}-\mathrm{Ni}$ are generally quite small in magnitude, and hence the neglect of all of the small but much larger number of further-neighbor solute-solute interactions is not accurate; again this can be computed and the agreement with simulations improved, but we do not present the details here.

Summarizing the analysis in this section, we have developed an analytic prediction for the standard deviation of the end-state energy difference $\Delta E_{\text {end }}$ in terms only of measurable solute/dislocation and solute/solute interaction energies. Explicitly combining the above results, the analysis yields

$$
\begin{aligned}
\sigma\left[\Delta E_{\mathrm{end}}\right]^{2} & =c(1-c) \frac{\zeta_{\mathrm{csn}}}{b} \sum_{i j}\left[U\left(x_{i}^{\prime}, y_{j}^{\prime}\right)-U\left(x_{i}, y_{j}\right)\right]^{2} \\
& +E_{\mathrm{s}-\mathrm{s}}^{2}\left(8 c^{2}(1-c) \frac{\zeta_{\mathrm{csn}} d}{b^{2} \sqrt{3}}\right) .
\end{aligned}
$$

This result, while validated against simulations for the current set of alloys and concentrations, is completely general. It can thus be applied to obtain the statistical distribution of $\Delta E_{\text {end }}$ relevant for the subsequent computation of cross-slip activation barriers for any other FCC alloys, whether existing or proposed alloys.

\section{Discussion}

We have shown that (i) the cross-slip activation energy $\Delta E_{\text {act }}$ in random fcc solid solutions is a stochastic variable due to the explicit variations in solute positions relative to the dislocation undergoing cross-slip, with large variations around the average cross-slip barrier; (ii) there is a linear correlation between $\Delta E_{\mathrm{act}}$ and the difference $\Delta E_{\text {end }}$ between the initial and final cross-slip states; (iii) the standard deviation of $\Delta E_{\text {end }}$ can be computed analytically as a function of alloy concentration, solute/dislocation interaction energies, solute-solute interaction energies, for the characteristic cross-slip nucleation length $\zeta_{\mathrm{csn}}$; and therefore (iv) we have an analytic model for the distribution of cross-slip activation energies in random fcc alloys. We now discuss various implications of these results.

First, average material properties are insufficient for predicting the rate of cross-slip in a real alloy. As noted in Sec. 3.3, low values of $\Delta E_{\text {act }}$ caused by random solute fluctuations will control the rate. In particular, an average parameter like the normalized stacking fault energy $\gamma_{\mathrm{s}} / \mu b$ is not a useful measure for the ease of cross-slip in random alloys. This conclusion may explain observations by Clement and Coulomb [97] in Ni-Cr. They measured the activation energy and apparent activation volume for cross-slip in $\mathrm{Ni}-\mathrm{Cr}$ alloys and found that these values decrease with increasing $\mathrm{Cr}$ content whereas an increase would be expected based on average properties because both $\gamma_{\mathrm{sf}}$ and $\gamma_{\mathrm{sf}} / \mu$ decrease with increasing $\mathrm{Cr}$ content.

Another consequence of the strong influence of random fluctuations on the activation barrier is that the probability of cross-slip nucleation in an alloy depends more strongly on the 
This is a post-print of the following article: W. G. Noehring; W. A. Curtin Acta Materialia 2017,, 14. 135-148.. The formal publication is available at http://dx.doi.org/10.1016/j.actamat.2017.02.027 (c) 2017. This manuscript version is made available under the CC-BY-NC-ND 4.0 license http://creativecommons.org/licenses/by-nc-nd/4.0/

length of the dislocation. Here, we must clearly distinguish between nucleation, i.e. cross-slip of a short (length $\zeta_{c s n}$ ) segment, and lateral growth of this segment. A dislocation line with a length of $\zeta$ consists of $N=\zeta / \zeta_{\text {csn }}$ segments of length $\zeta_{\text {csn }}$. A long dislocation with large $N$ will sample many different local solute configurations and hence many local cross-slip nucleation environments and is therefore likely to contain segments with cross-slip energy barriers that are much lower than the average value. Among the $N$ segments, the segment with the lowest activation barrier satisfies, on average,

$$
\int_{-\infty}^{\Delta E_{\mathrm{act}}^{*}} P\left(\Delta E_{\mathrm{act}}^{\prime}\right) d \Delta E_{\mathrm{act}}^{\prime}=\frac{1}{N}
$$

where $\mathrm{P}\left(\Delta E_{\text {act }}\right)$ is the Gaussian distribution of activation energies Eq. 7. Thus, $\Delta E_{\text {act }}^{*}$ can be obtained from the inverse normal cumulative distribution function. An asymptotic approximation for large $N$ [98] yields

$$
\Delta E_{\mathrm{act}}^{*} \approx \Delta E_{\mathrm{act}, \mathrm{avg}}-\frac{\sigma}{2} \sqrt{\log \left(\frac{N^{2}}{2 \pi}\right)-\log \left(\log \left(\frac{N^{2}}{2 \pi}\right)\right)}
$$

The weak dependence of the smallest activation energy on the number of segments $N$ allows for reasonable estimates of the barrier over a wide range of lengths. Typical dislocation densities in metals range from well-annealed materials with dislocation density $\rho=10^{11}-10^{12} \mathrm{~m}^{-2}$ to highly deformed materials with $\rho=10^{14} \mathrm{~m}^{-2}$. The corresponding dislocation segment lengths are $\zeta \propto 1 / \sqrt{\rho}$ and thus vary from $100 \mathrm{~nm}$ to $3000 \mathrm{~nm}$. For $\zeta_{\mathrm{csn}}=40 \mathrm{~b}$ and $\rho \approx 10^{12} \mathrm{~m}^{-2}$, the minimum activation barriers for such segment lengths are $\Delta E_{\text {act }} \approx \Delta E_{\text {act,avg }}-1.54 \sigma\left[\Delta E_{\text {end }}\right]$. For a typical Al-2 at.\% Mg alloy, this activation energy is only $0.42 \mathrm{eV}$. For a typical Ni-10 at.\% Al alloy (model matrix for Ni$\mathrm{Al}$ superalloys), the operative barrier would be $0.55 \mathrm{eV}$. For a $\mathrm{Cu}$ 33 at.\% Ni alloy, the operative barrier would be $1.06 \mathrm{eV}$. All of these energy barriers are significantly below the average energy barrier of the same alloy, and correspond to very large increases in cross-slip rate at moderate temperatures (for instance, see Eq. 1 with $k T=0.0254 \mathrm{eV}$ at $T=300 \mathrm{~K}$ ).

The barriers for cross-slip nucleation can therefore be very low in Ni-Al and $\mathrm{Al}-\mathrm{Mg}$. However, a nucleus of length $40 \mathrm{~b}$ must subsequently expand along the dislocation line by lateral motion of the two constrictions in order to grow the cross-slip region. Expansion involves sequentially overcoming smaller energy changes of the order of $\sigma\left[\Delta E_{\text {end }}(\zeta=1 b)\right]$ for the lateral advance of the cross-slipped segment by one Burgers vector. These energy changes can be positive or negative, depending on whether the glide or cross-slip plane is energetically favorable at each site. For a long dislocation line, the growing nucleus will eventually encounter regions where there is a long sequence of small positive energy changes, and this sequence can create a significant barrier against further lateral expansion of the cross-slip segment nucleation. On the other hand, if the nucleated segment can expand sufficiently before encountering such a barrier, it can still activate the myriad processes associated with cross-slip. That is, extension of the cross-slip across the entire screw-oriented portion of the line length is not necessary to create cross-slip processes in the alloy. The analysis of characteristic cross-slip segment lengths, and barriers, is a statistical problem that is beyond the scope of this paper. The discussion here serves to identify the problem, and to emphasize that while cross-slip is not driven the average barrier, neither is it driven completely by the single lowest barrier (weak-link behavior). We will address this statistical problem, and its important implications for experimental observation of cross-slip in alloys, in future work.

The present analysis has identified some crucial new features of cross-slip activation in random fcc alloys, and developed an analytic predictive model that captures the controlling statistical aspects of the problem. The concepts and analysis apply to both dilute and concentrated solid solutions. The latter category includes High Entropy Alloys, where the effects identified here may provide insight into the origins of the strength and work hardening behavior. However, the full implications of our results have not yet been explored. In addition, the role of Escaig stresses, i.e. applied stress that change the partial dislocation separation and thus the cross-slip barrier, have not yet been investigated. We are currently studying both of these important aspects of cross-slip and will report on our analysis in the near future.

\section{Acknowledgment}

Support for this work was provided by the European Research Council through the Advanced Grant "Predictive Computational Metallurgy", ERC Grant agreement No. 339081 - PreCoMet. Furthermore, support was provided by EPFL through the use of the facilities of its Scientific IT and Application Support Center. WGN thanks Dr. Z. Wu for helpful discussions during the course of this work.

\section{Appendix A. Implementation of the Simplified String Method}

The transition path is a path on the (3N-1)-dimensional potential energy landscape, where $\mathrm{N}$ is the number of atoms. It connects the initial and final state of the process and its characteristic is that a state lying on it experiences no force perpendicular to the path.

In a numerical scheme, the path is discretized using a number of $N_{S}$ "images" of the system of interest, which includes the initial and the final state. The images are placed along the path according to a specific parameterization. Starting from an initial guess, the solution is found iteratively.

LAMMPS implements the Nudged Elastic Band [71] method for transition path calculation. However, this method diverged frequently in preliminary calculations with $\mathrm{Ni}-\mathrm{Al}$ and $\mathrm{Al}-\mathrm{Mg}$. As an alternative, we used a custom implementation of the Simplified and Improved String [58] method.

Each iteration consists two steps: an evolution step, where the atoms are moved according to their force, and an interpolation step, which is required to retain the parameterization. In our implementation, we require the images to be equally spaced along the path. FIRE energy minimization is used for moving the atoms, and linear interpolation for reparameterization. 
This is a post-print of the following article: W. G. Noehring; W. A. Curtin Acta Materialia 2017,, 14. 135-148.. The formal publication is available at http://dx.doi.org/10.1016/j.actamat.2017.02.027 (C) 2017. This manuscript version is made available under the CC-BY-NC-ND 4.0 license http://creativecommons.org/licenses/by-nc-nd/4.0/

The used FIRE parameters are listed in Tab. A.1. E et al. [58] reparameterized the path after every increment of the motion. Considering the large $\left(\propto 10^{5}\right)$ number of degrees of freedom in our calculation, we reparameterize only every 100 increments of the minimizer, which is similar to using a larger overall step size. Iteration is stopped when the norm of the displacement of each image relative to its position in the previous iteration is below $10^{-3} \AA$, or, if this threshold is not reached, after a total number of 300 iterations.

Table A.1: FIRE parameters; see [84] for reference.

\begin{tabular}{cc}
\hline parameter & value \\
\hline$N_{\min }$ & 20 \\
$f_{\text {inc }}$ & 1.1 \\
$f_{\text {dec }}$ & 0.5 \\
$f_{\alpha}$ & 0.99 \\
$\alpha_{\text {start }}$ & 0.25 \\
$\Delta t_{\text {max }}$ & $0.002 \mathrm{ps}$ \\
$\Delta t$ (initial) & $0.001 \mathrm{ps}$ \\
\hline
\end{tabular}

Note that in the original implementation of the Modified Improved String method, the atoms would be moved according to the following overdamped dynamics:

$$
\dot{\mathbf{x}}_{i}=-\Delta V_{i}, \quad\left(i=1 \ldots N_{S}\right),
$$

where $\dot{\mathbf{x}}_{i}$ is the $3 N$-dimensional vector of atomic velocities of image $i$. The idea is that all images lying on the path represents a stationary state of the whole scheme, since images on the path will only slide downhill along the path and will be moved back to their original position in the subsequent interpolation step. In our implementation, by contrast, the atoms are moved according to FIRE dynamics, see [84]. This is Newtonian dynamics, where, however, the velocity and force vector are mixed to obtain a velocity with a stronger downhill component. Furthermore, velocities are zeroed whenever the dot product of velocity and momentum is positive, i.e. whenever the system is moving uphill on the energy landscape. In conjunction, this means that states on the path will only move downhill along the path, like in the original scheme; i.e. all states lying on the path is a stationary state of the modified scheme, too.

\section{Appendix B. Solute pair model}

Dislocation cross-slip involves annihilation of the stacking fault ribbon on the glide plane and formation of a new one on the cross-slip plane. In each fault ribbon, two atomic layers are displaced by one partial Burgers vector relative to each other. As a consequence, solute-solute nearest-neighbor pairs may be formed or destroyed. The net change in solute pairs $\Delta N_{\mathrm{s}-\mathrm{s}}$ during cross-slip is a random variable. We want to model its standard deviation.

We make the simplifying assumption that the dislocations are straight and the stacking fault is an ideal stacking fault everywhere. In reality, the dislocations may be curved and the atomic displacement close to the partial dislocation cores that delimit the fault is not exactly the displacement corresponding to a perfect fault. Furthermore, we assume that the solute distribution is random and uncorrelated, so that we can replace occupation numbers by the average solute concentration.

Fig. B.10 shows the two atomic layers in a section of a stacking fault. Atoms in the lower layer are gray. A crystallographic unit cell is shaded red. In a stacking fault ribbon of length $\zeta$ along $[10 \overline{1}]$, the dislocation line direction, and width $d$ along $[1 \overline{2} 1]$, there are $\zeta / b \times d / b \sqrt{3}$ such cells. In each cell, there are two atoms in the upper layer. Each atom exchanges one nearest neighbor in the lower plane when the fault is formed or annihilated.

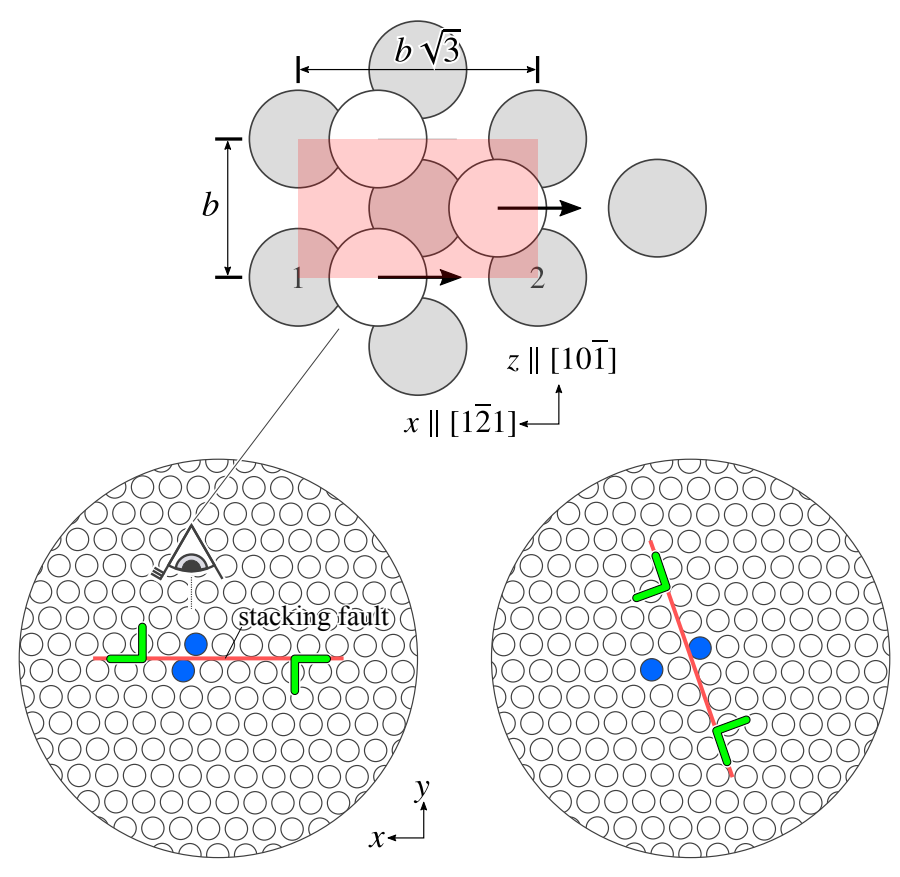

Figure B.10: Lower images: layer of atoms perpendicular to the dislocation line; a nearest-neighbor pair of solutes (blue) in the stacking fault (red) is destroyed as the dislocation moves from the glide to the cross-slip plane. Top: view from top onto the stacking fault; gray atoms are in the lower layer of the fault. Per unit cell in the fault (shaded red), there are two atoms in the upper layer of the fault, each of which exchanges one nearest neighbor atom in the lower layer during cross-slip.

Let $N_{\zeta} \equiv 2 \zeta / b$ and $N_{d} \equiv d / b \sqrt{3}$. Label the atoms in the upper layer by $i=1 \ldots N_{\zeta}$ along $x \|[10 \overline{1}]$ and by $j=1 \ldots N_{d}$ along $y \|[1 \overline{2} 1]$. The net change in solute-solute pairs when the fault in Fig. B.10 is formed or destroyed is:

$$
\Delta N_{\mathrm{s}-\mathrm{s}, \mathrm{sf}}=\sum_{i}^{N_{l}} \sum_{j}^{N_{d}} s_{i j}\left(s_{i j, 2}-s_{i j, 1}\right),
$$

where $s_{i j}, s_{i j, 1}$ and $s_{i j, 2}$ are the occupation numbers of the atom at site $(i, j)$ and the two nearest neighbors in the lower plane that are exchanged in the process. $s_{i j}$ is one if there is a solute at site $(i, j)$, and zero otherwise. The same applies to $s_{i j, 1}$ and $s_{i j, 2}$. For convenience, let $u_{i j} \equiv\left(s_{i j, 2}-s_{i j, 1}\right)$. Note that $\left\langle u_{p q}\right\rangle=c-c=$ $0 \forall p, q$.

Calculating the standard deviation of $\Delta N_{\mathrm{s}-\mathrm{s} \text {,sf }}$ requires $\left\langle\Delta N_{\mathrm{s}-\mathrm{s}, \mathrm{sf}}\right\rangle^{2}$ 
This is a post-print of the following article: W. G. Noehring; W. A. Curtin Acta Materialia 2017,, 14. 135-148.. The formal publication is available at http://dx.doi.org/10.1016/j.actamat.2017.02.027 (C) 2017. This manuscript version is made available under the CC-BY-NC-ND 4.0 license http://creativecommons.org/licenses/by-nc-nd/4.0/

and $\left\langle\Delta N_{\mathrm{s}-\mathrm{s}, \mathrm{sf}}{ }^{2}\right\rangle$. The former is zero, because

$$
\begin{aligned}
\left\langle\Delta N_{\mathrm{s}-\mathrm{s}, \mathrm{sf}}\right\rangle & =\sum_{i}^{N_{l}} \sum_{j}^{N_{d}}\left\langle s_{i j}\left(s_{i j, 2}-s_{i j, 1}\right)\right\rangle \\
& =\sum_{i}^{N_{l}} \sum_{j}^{N_{d}}\left\langle s_{i j}\right\rangle\left(\left\langle s_{i j, 2}\right\rangle-\left\langle s_{i j, 1}\right\rangle\right) \\
& =\sum_{i}^{N_{l}} \sum_{j}^{N_{d}} c(c-c) \\
& =0 .
\end{aligned}
$$

Furthermore,

$$
\begin{aligned}
\Delta N_{\mathrm{s}-\mathrm{s}, \mathrm{sf}}^{2} & =\left[\sum_{i}^{N_{l}} \sum_{j}^{N_{d}} s_{i j} u_{i j}\right]^{2} \\
& =\sum_{i}^{N_{l}} \sum_{j}^{N_{d}}\left(s_{i j} u_{i j}\right)^{2} \\
& +\sum_{i}^{N_{l}} \sum_{j}^{N_{d}} \sum_{k \neq j}^{N_{d}} s_{i j} u_{i j} s_{i k} u_{i k}+\sum_{i}^{N_{l}} \sum_{k \neq i}^{N_{l}} \sum_{j}^{N_{d}} s_{i j} u_{i j} s_{k j} u_{k j} \\
& +\sum_{i}^{N_{l}} \sum_{k \neq i}^{N_{l}} \sum_{j}^{N_{d}} \sum_{l \neq j}^{N_{d}} s_{i j} u_{i j} s_{k l} u_{k l} .
\end{aligned}
$$

Averaging eliminates all but the first term, therefore:

$$
\left\langle\Delta N_{\mathrm{s}-\mathrm{s}, \mathrm{sf}}^{2}\right\rangle \quad=\sum_{i} \sum_{j}^{N_{l}}\left\langle s_{i j}^{2}\right\rangle\left\langle u_{i j}^{2}\right\rangle .
$$

$s_{i j}, s_{i j, 1}$ and $s_{i j, 2}$ are Bernoulli random variables, hence:

$$
\begin{aligned}
\left\langle u_{i j}^{2}\right\rangle & =\left\langle s_{i j, 2}^{2}-2 s_{i j, 2} s_{i j, 1}+s_{i j, 1}^{2}\right\rangle \\
& =\left\langle s_{i j, 2}^{2}\right\rangle-2\left\langle s_{i j, 2} s_{i j, 1}\right\rangle+\left\langle s_{i j, 1}^{2}\right\rangle \\
& =c-2 c^{2}+c \\
& =2 c(1-c) .
\end{aligned}
$$

Thus

$$
\begin{aligned}
\left\langle\Delta N_{\mathrm{s}-\mathrm{s}, \mathrm{sf}}{ }^{2}\right\rangle & =\sum_{i}^{N_{l}} \sum_{j}^{N_{d}} 2 c^{2}(1-c) \\
& =2 c^{2}(1-c) N_{l} N_{d} \\
& =4 c^{2}(1-c) \frac{\zeta d}{b^{2} \sqrt{3}} .
\end{aligned}
$$

Assuming that pair formation and destruction on the glide and cross-slip plane is independent, the variance of the net pair change due to cross-slip is:

$$
\operatorname{Var}\left[\Delta N_{\mathrm{s}-\mathrm{s}}\right]=2\left(\left\langle\Delta N_{\mathrm{s}-\mathrm{s}, \mathrm{sf}}^{2}\right\rangle-\left\langle\Delta N_{\mathrm{s}-\mathrm{s}, \mathrm{sf}}\right\rangle^{2}\right) .
$$

The standard deviation is therefore:

$$
\sigma\left[\Delta N_{\mathrm{s}-\mathrm{s}}\right]=\sqrt{8 c^{2}(1-c) \frac{\zeta d}{b^{2} \sqrt{3}}} .
$$

\section{References}

[1] N. F. Mott, A theory of the origin of fatigue cracks, Acta Metallurgica 6 (3) (1958) 195-197. doi: $10.1016 / 0001-6160$ (58) 90007-5.

[2] J. S. Koehler, The nature of work-hardening, Physical Review 86 (1) (1952) 52-59. doi:10.1103/PhysRev.86.52.

[3] P. Hirsch, The interpretation of the slip pattern in terms of dislocation movements, Journal of the Institute of Metals 86 (1957) 13.

[4] C. Singh, A. Mateos, D. Warner, Atomistic simulations of dislocationprecipitate interactions emphasize importance of cross-slip, Scripta Materialia 64 (5) (2011) 398-401. doi :10.1016/j.scriptamat. 2010 10.041 .

[5] G. Schoeck, A. Seeger, Activation Energy Problems associated with Extended Dislocations, in: Report of a Conference on Defects in Crystalline Solids, Physical Society, London, 1955, pp. 340-346.

[6] H. Wolf, Die Aktivierungsenergie für die Quergleitung aufgespaltener Schraubenversetzungen, Zeitschrift für Naturforschung 15A (1960) 180 193.

[7] J. Friedel, Regarding Seeger's Paper on Work Hardening, in: J. Fisher, W. Johnston, R. Thomson, T. Vreeland, Jr. (Eds.), Dislocations and Mechanical Properties of Crystals, John Wiley \& Sons, Inc., 1957, pp. 330 332.

[8] B. Escaig, Sur le glissement dévié des dislocations dans la structure cubique à faces centrées, Journal de Physique 29 (2-3) (1968) 225-239. doi:10.1051/jphys:01968002902-3022500.

[9] J. Washburn, Intersection Cross Slip, Applied Physics Letters 7 (7) (1965) 183. doi:10.1063/1.1754369.

[10] R. L. Fleischer, Cross slip of extended dislocations, Acta Metallurgica 7 (2) (1959) 134-135. doi:10.1016/0001-6160 (59) 90122-1.

[11] M. Marcinkowski, K. Sadananda, N. J. Olson, Cross Slip of Extended Dislocations in Disordered Face-Centred-Cubic Alloys, Crystal Lattice Defects 5 (1974) 187-198

[12] G. Vanderschaeve, Cross-Slip of Partial Dislocations via the Stair Rod Mode, physica status solidi (a) 100 (59) (1987) 59-68. doi : $10.1002 /$ pssa.2211000107.

[13] J. Bonneville, B. Escaig, Cross-Slipping Process and the Stress-Orientation Dependence in Pure Copper, Acta Metallurgica 27 (1979) 1477-1486. doi:10.1016/0001-6160(79) 90170-6.

[14] M. S. Duesbery, N. P. Louat, K. Sadananda, The mechanics and energetics of cross-slip, Acta metallurgica et materialia 40 (1) (1992) 149-158. doi: $10.1016 / 0956-7151$ (92) 90208-V.

[15] W. Püschl, G. Schoeck, Calculation of cross-slip parameters in fcc crystals, Materials Science and Engineering: A 164 (1) (1993) 286-289. doi : 10.1016/0921-5093 (93) 90679-9.

[16] B. R. Ramírez, N. Ghoniem, G. Po, Ab initio continuum model for the influence of local stress on cross-slip of screw dislocations in fcc metals, Physical Review B 86 (9). doi:10.1103/PhysRevB.86.094115.

[17] K. Kang, J. Yin, W. Cai, Stress dependence of cross slip energy barrier for face-centered cubic nickel, Journal of the Mechanics and Physics of Solids 62 (2014) 181-193. doi:10.1016/j.jmps.2013.09.023.

[18] G. Saada, Cross-slip and work hardening of fcc crystals, Materials Science and Engineering: A 137 (1991) 177-183. doi:10.1016/ 0921-5093 (91) 90333-I.

[19] T. Rasmussen, K. W. Jacobsen, T. Leffers, O. B. Pedersen, Simulations of the atomic structure, energetics, and cross slip of screw dislocations in copper, Physical Review B 56 (6) (1997) 2977. doi:10.1103/ PhysRevB. 56.2977.

[20] T. Vegge, T. Rasmussen, T. Leffers, O. B. Pedersen, K. W. Jacobsen, Determination of the of rate cross slip of screw dislocations, Physical review letters 85 (18) (2000) 3866. doi:10.1103/PhysRevLett. 85.3866.

[21] S. Rao, T. A. Parthasarathy, C. Woodward, Atomistic simulation of crossslip processes in model fcc structures, Philosophical Magazine A 79 (5) (1999) 1167-1192. doi:10.1080/01418619908210354.

[22] S. Rao, D. Dimiduk, J. El-Awady, T. Parthasarathy, M. Uchic, C. Woodward, Screw dislocation cross slip at cross-slip plane jogs and screw dipole annihilation in FCC Cu and Ni investigated via atomistic simulations, Acta Materialia 101 (2015) 10-15. doi :10.1016/j.actamat.2015.08. 070 .

[23] A. M. Hussein, S. I. Rao, M. D. Uchic, D. M. Dimiduk, J. A. El-Awady, Microstructurally based cross-slip mechanisms and their effects on dislo- 
This is a post-print of the following article: W. G. Noehring; W. A. Curtin Acta Materialia 2017,, 14. 135-148.. The formal publication is available at http://dx.doi.org/10.1016/j.actamat.2017.02.027 ( ) 2017. This manuscript version is made available under the CC-BY-NC-ND 4.0 license http://creativecommons.org/licenses/by-nc-nd/4.0/

cation microstructure evolution in fcc crystals, Acta Materialia 85 (2015) 180-190. doi:10.1016/j.actamat.2014.10.067.

[24] E. Bitzek, C. Brandl, P. Derlet, H. Van Swygenhoven, Dislocation CrossSlip in Nanocrystalline fcc Metals, Physical Review Letters 100 (23) doi:10.1103/PhysRevLett.100.235501.

[25] M. S. Duesbery, Dislocation motion, constriction and cross-slip in fcc metals, Modelling and Simulation in Materials Science and Engineering 6 (1) (1998) 35. doi : 10.1088/0965-0393/6/1/005.

[26] C. Jin, Y. Xiang, G. Lu, Dislocation cross-slip mechanisms in aluminum, Philosophical Magazine 91 (32) (2011) 4109-4125. doi:10.1080/ 14786435.2011 .602030

[27] S. Pendurti, S. Jun, I.-H. Lee, V. Prasad, Cooperative atomic motions and core rearrangement in dislocation cross slip, Applied Physics Letters 88 (20) (2006) 201908. doi:10.1063/1.2204449.

[28] M. Cawkwell, C. Woodward, D. Nguyenmanh, D. Pettifor, V. Vitek, Atomistic study of athermal cross-slip and its impact on the mechanical properties of iridium, Acta Materialia 55 (1) (2007) 161-169. doi : $10.1016 / j$.actamat. 2006.07 .033 .

[29] U. Essmann, Elektronenmikroskopische Untersuchung der Versetzungsanordnung in plastisch verformten Kupfereinkristallen, Acta Metallurgica 12 (12) (1964) 1468-1470. doi:10.1016/0001-6160 (64) $90142-7$.

[30] Z. S. Basinski, Work hardening: Surface effects. Dislocation distribution in deformed copper single crystals, Discussions of the Faraday Society 38 (1964) 93-102. doi:10.1039/DF 9643800093.

[31] J. W. Steeds, P. Hazzledine, Dislocation configurations in deformed copper and copper $10 \%$ (atomic) aluminium alloy, Discussions of the Faraday Society 38 (1964) 103-110. doi:10.1039/DF 9643800103.

[32] P. J. Jackson, The role of cross-slip in the plastic deformation of crystals, Materials Science and Engineering 57 (1) (1983) 39-47. doi : $10.1016 /$ $0025-5416$ (83) 90025-3.

[33] U. Essmann, H. Mughrabi, Annihilation of dislocations during tensile and cyclic deformation and limits of dislocation densities, Philosophical Magazine A 40 (6) (1979) 731-756. doi:10.1080/ 01418617908234871.

[34] S. Xia, J. Belak, A. El-Azab, The discrete-continuum connection in dislocation dynamics: I. Time coarse graining of cross slip, Modelling and Simulation in Materials Science and Engineering 24 (7) (2016) 075007. doi:10.1088/0965-0393/24/7/075007.

[35] U. F. Kocks, Laws for Work-Hardening and Low-Temperature Creep, Journal of Engineering Materials and Technology 98 (1) (1976) 76-85. doi:10.1115/1.3443340.

[36] W. D. Nix, J. C. Gibeling, D. A. Hughes, Time-dependent deformation of metals, Metallurgical Transactions A 16 (12) (1985) 2215-2226. doi : $10.1007 / \mathrm{BF} 02670420$.

[37] K. Karhausen, F. Roters, Development and application of constitutive equations for the multiple-stand hot rolling of Al-alloys, Journal of Materials Processing Technology 123 (2002) 155-166. doi:10.1016/ S0924-0136(02) 00081-X.

[38] J. Dorn, N. Jaffe, Effect of Temperature on the Creep of Polycrystalline Aluminum by the Cross-Slip Mechanism, Transactions of the Metallurgical Society of AIME 221 (1961) 229-233.

[39] J. Lytton, L. Shepard, J. Dorn, The Activation Energies for Creep of Single Aluminum Crystals Favorably Oriented for (III)[T0I] Slip, Transactions of the American Institute of Mining, Metallurgical and Petroleum Engineers 212 (1958) 220

[40] J. Friedel, Sur le fluage par déviation, Revue de Physique Appliquée 12 (10) (1977) 1649-1654. doi:10.1051/rphysap: 0197700120100164900.

[41] J. Poirier, On the symmetrical role of cross-slip of screw dislocations and climb of edge dislocations as recovery processes controlling hightemperature creep, Revue de Physique Appliquée 11 (6) (1976) 731-738. doi:10.1051/rphysap:01976001106073100.

[42] O. Sherby, J. R. Weertman, Diffusion-Controlled Dislocation Creep: A Defense, Acta Metallurgica 27 (1979) 387-400. doi:10.1016/ $0001-6160$ (79) $90031-2$.

[43] J.-P. Poirier, Reply to "Diffusion-Controlled Dislocation Creep: A Defense", Acta Metallurgica 27 (1979) 401-403. doi:10.1016/ $0001-6160$ (79) $90032-4$

[44] S. V. Raj, Power-law and exponential creep in class M materials: Discrepancies in experimental observations and implications for creep mod- eling, Materials Science and Engineering: A 322 (1) (2002) 132-147. doi:10.1016/0001-6160(79)90032-4.

[45] R. E. Smallman, D. Green, The dependence of rolling texture on stacking fault energy, Acta Metallurgica 12 (2) (1964) 145-154. doi: $10.1016 /$ $0001-6160$ (64) $90182-8$.

[46] I. L. Dillamore, W. Roberts, Rolling Textures in F.C.C. and B.C.C. Metals, Acta Metallurgica 12 (3) (1964) 281-293. doi:10.1016/ $0001-6160$ (64) 90204-4.

[47] T. Leffers, O. B. Pedersen, The activation energy for the fcc rolling-texture transition as related to the activation energy for cross slip, Scripta materialia 46 (10) (2002) 741-746. doi : $10.1016 /$ S1359-6462 (02) $00065-9$.

[48] T. Leffers, R. Ray, The brass-type texture and its deviation from the copper-type texture, Progress in Materials Science 54 (3) (2009) 351-396. doi:10.1016/j.pmatsci.2008.09.002.

[49] A. Seeger, The Mechanism of Glide and Work Hardening in Face-Centered Cubic and Hexagonal Close-Packed Metals, in: J. Fisher, W. Johnston, R. Thomson, T. Vreeland, Jr. (Eds.), Dislocations and Mechanical Properties of Crystals, John Wiley \& Sons, Inc., 1957, pp. 243-329.

[50] L. Kubin, T. Hoc, B. Devincre, Dynamic recovery and its orientation dependence in face-centered cubic crystals, Acta Materialia 57 (8) (2009) 2567-2575. doi:10.1016/j.actamat.2009.02.013.

[51] A. S. Argon, A new model of dynamic recovery for Stage III of pure fcc metals without cross slip, International Journal of Materials Research 100 (4) (2009) 488-493. doi : 10 .3139/146.110059.

[52] D. Caillard, J. Martin, Thermally Activated Mechanisms in Crystal Plasticity, 1st Edition, Vol. 8 of Pergamon Materials Series, Pergamon, Amsterdam, 2003.

[53] R. Heidenreich, W. Shockley, Study of Slip in Aluminium Crystals by Electron Microscope and Electron Diffraction Methods, in: Report of a Conference on Strength of Solids, Physical Society, London, 1948, pp. 57-75.

[54] A. N. Stroh, Constrictions and jogs in extended dislocations, Proceedings of the Physical Society. Section B 67 (5) (1954) 427. doi: $10.1088 /$ $0370-1301 / 67 / 5 / 307$.

[55] W. Püschl, Models for dislocation cross-slip in close-packed crystal structures: A critical review, Progress in materials science 47 (4) (2002) 415461. doi:10.1016/S0079-6425(01)00003-2.

[56] E. Martínez, J. Marian, A. Arsenlis, M. Victoria, J. Perlado, Atomistically informed dislocation dynamics in fcc crystals, Journal of the Mechanics and Physics of Solids 56 (3) (2008) 869-895. doi : $10.1016 / j$. jmps . 2007.06 .014$.

[57] T. Rasmussen, K. W. Jacobsen, T. Leffers, O. B. Pedersen, S. G. Srinivasan, H. Jonsson, Atomistic determination of cross-slip pathway and energetics, Physical review letters 79 (19) (1997) 3676. doi: $10.1103 /$ PhysRevLett. 79.3676.

[58] W. E, W. Ren, E. Vanden-Eijnden, Simplified and improved string method for computing the minimum energy paths in barrier-crossing events, The Journal of Chemical Physics 126 (16) (2007) 164103. doi: $10.1063 /$ 1. 2720838 .

[59] J. D. Honeycutt, H. C. Andersen, Molecular Dynamics Study of Melting and Freezing of Small Lennard-Jones Clusters, Journal of Physical Chemistry 91 (1987) 4950-4963. doi : 10.1021 / j100303a014.

[60] A. Stukowski, Visualization and analysis of atomistic simulation data with OVITO-the Open Visualization Tool, Modelling and Simulation in Materials Science and Engineering 18 (1) (2010) 015012. doi : 10 1088/0965-0393/18/1/015012.

[61] B. Escaig, Cross-Slipping Process in the F.C.C. Structure, in: A. Rosenfield, G. Hahn, A. Bement Jr., R. Jaffee (Eds.), Proceedings of the Battelle Colloquium on Dislocation Dynamics, McGraw-Hill, 1968, pp. 655-677.

[62] T. Vegge, T. Rasmussen, T. Leffers, O. Pedersen, K. Jacobsen, Atomistic simulations of cross-slip of jogged screw dislocations in copper, Philosophical Magazine Letters 81 (3) (2001) 137-144. doi:10.1080/ 09500830010019040 .

[63] S. Rao, D. Dimiduk, J. El-Awady, T. Parthasarathy, M. Uchic, C. Woodward, Atomistic simulations of cross-slip nucleation at screw dislocation intersections in face-centered cubic nickel, Philosophical Magazine 89 (3436) (2009) 3351-3369. doi : $10.1080 / 14786430903286201$

[64] S. Rao, D. Dimiduk, J. El-Awady, T. Parthasarathy, M. Uchic, C. Woodward, Activated states for cross-slip at screw dislocation intersections in face-centered cubic nickel and copper via atomistic simulation, Acta Mate- 
This is a post-print of the following article: W. G. Noehring; W. A. Curtin Acta Materialia 2017,, 14. 135-148.. The formal publication is available at http://dx.doi.org/10.1016/j.actamat.2017.02.027 (C) 2017. This manuscript version is made available under the CC-BY-NC-ND 4.0 license http://creativecommons.org/licenses/by-nc-nd/4.0/

rialia 58 (17) (2010) 5547-5557. doi:10.1016/j.actamat.2010 06.005

[65] S. Rao, D. Dimiduk, J. El-Awady, T. Parthasarathy, M. Uchic, C. Woodward, Spontaneous athermal cross-slip nucleation at screw dislocation intersections in FCC metals and $\mathrm{L} 12$ intermetallics investigated via atomistic simulations, Philosophical Magazine 93 (22) (2013) 3012-3028. doi:10.1080/14786435.2013.799788.

[66] S. Rao, D. Dimiduk, T. Parthasarathy, J. El-Awady, C. Woodward, M. Uchic, Calculations of intersection cross-slip activation energies in fcc metals using nudged elastic band method, Acta Materialia 59 (19) (2011) 7135-7144. doi:10.1016/j.actamat.2011.08.029.

[67] S. Rao, D. Dimiduk, T. Parthasarathy, M. Uchic, C. Woodward, Atomistic simulations of surface cross-slip nucleation in face-centered cubic nickel and copper, Acta Materialia 61 (7) (2013) 2500-2508. doi:10.1016/ j.act amat. 2013.01 .026$.

[68] P. Coulomb, Comment on graphs relating some property to stacking fault energy, Scripta Metallurgica 15 (7) (1981) 769-770. doi:10.1016/ 0036-9748 (81) 90017-X.

[69] S. D. Andrews, H. Sehitoglu, I. Karaman, Constriction energy in the presence of a solute field, Journal of Applied Physics 87 (5) (2000) 2194 doi:10.1063/1.372161.

[70] J.-P. Du, C.-Y. Wang, T. Yu, Cross-slip process in model Ni(Al) solid solution: An embedded-atom method study, Computational Materials Science 91 (2014) 192-199. doi:10.1016/j.commatsci.2014. 04.063

[71] G. Mills, H. Jónsson, Quantum and thermal effects in H 2 dissociative adsorption: Evaluation of free energy barriers in multidimensional quantum systems, Physical review letters 72 (7) (1994) 1124. doi: 10.1103/PhysRevLett. 72.1124.

[72] M. Wen, S. Fukuyama, K. Yokogawa, Hydrogen-affected cross-slip process in fcc nickel, Physical Review B 69 (17). doi:10.1103/ PhysRevB.69.174108.

[73] M. Wen, S. Fukuyama, K. Yokogawa, Cross-slip process in fcc nickel with hydrogen in a stacking fault: An atomistic study using the embedded-atom method, Physical Review B 75 (14). doi:10.1103/PhysRevB. 75. 144110 .

[74] X.-Y. Liu, J. B. Adams, F. Ercolessi, J. A. Moriarty, EAM potential for magnesium from quantum mechanical forces, Modelling and Simulation in Materials Science and Engineering 4 (1996) 293-303. doi:10.1088/ 0965-0393/4/3/004

[75] G. Purja Pun, Y. Mishin, Development of an interatomic potential for the Ni-Al system, Philosophical Magazine 89 (34-36) (2009) 3245-3267. doi:10.1080/14786430903258184.

[76] B. Onat, S. Durukanoğlu, An optimized interatomic potential for $\mathrm{Cu}-\mathrm{Ni}$ alloys with the embedded-atom method, Journal of Physics: Condensed Matter 26 (3) (2014) 035404. doi:10.1088/0953-8984/26/3/ 035404

[77] J.-W. Yeh, S.-K. Chen, S.-J. Lin, J.-Y. Gan, T.-S. Chin, T.-T. Shun, C.-H. Tsau, S.-Y. Chang, Nanostructured High-Entropy Alloys with Multiple Principal Elements: Novel Alloy Design Concepts and Outcomes, Advanced Engineering Materials 6 (5) (2004) 299-303. doi: 10.1002 /adem. 200300567.

[78] R. W. Smith, G. Was, Molecular Dynamics Simulation of the Role of Grain Boundary Chemistry on Intergranuar Fracture, Materials Science Forum 46 (1989) 199-236. doi:10.4028/www.scientific.net/MSF 46.199 .

[79] C. Varvenne, A. Luque, W. G. Nöhring, W. A. Curtin, Average-atom interatomic potential for random alloys, Physical Review B 93 (10) (2016) 104201. doi:10.1103/PhysRevB.93.104201.

[80] M. S. Daw, M. Baskes, Embedded-atom method: Derivation and application to impurities, surfaces, and other defects in metals, Physical Review B 29 (12) (1984) 6443-6453. doi:10.1103/PhysRevB. 29.6443.

[81] W. G. Nöhring, W. A. Curtin, Thermodynamic properties of average-atom interatomic potentials for alloys, Modelling and Simulation in Materials Science and Engineering 24 (4) (2016) 045017. doi:10.1088/ 0965-0393/24/4/045017.

[82] A. Stroh, Dislocations and Cracks in Anisotropic Elasticity, Philosophical Magazine 3 (30) (1958) 625-646. doi:10.1080/ 14786435808565804.

[83] M. R. Hestenes, E. Stiefel, Methods of Conjugate Gradients for Solving Linear Systems, Journal of Research of the National Bureau of Standards
49 (6) (1952) 409-436. doi:10.6028/jres.049.044.

[84] E. Bitzek, P. Koskinen, F. Gähler, M. Moseler, P. Gumbsch, Structural Relaxation Made Simple, Physical Review Letters 97 (17). doi:10. 1103/PhysRevLett.97.170201.

[85] S. Plimpton, Fast Parallel Algorithms for Short-Range Molecular Dynamics, Journal of Computational Physics 117 (1995) 1-19. doi: 10.1006/jcph.1995.1039.

[86] H. Jónsson, G. Mills, K. W. Jacobsen, Nudged elastic band method for finding minimum energy paths of transitions, in: B. Berne, G. Cicotti, D. Coker (Eds.), Classical and Quantum Dynamics in Condensed Phase Simulations, World Scientific, 1998, pp. 385-404.

[87] G. S. Hammond, A Correlation of Reaction Rates, Journal of the American Chemical Society 77 (2) (1955) 334-338. doi:10.1021/ ja01607a027.

[88] R. P. Bell, The Theory of Reactions Involving Proton Transfers, Proceedings of the Royal Society of London 154 (1936) 414-429. doi: $10.1098 / \mathrm{rspa} .1936 .0060$.

[89] M. G. Evans, M. Polanyi, Further considerations on the thermodynamics of chemical equilibria and reaction rates, Transactions of the Faraday Society 32 (1936) 1333-1360. doi:10.1039/TF9363201333.

[90] K. A. Dill, S. Bromberg, Molecular Driving Forces, 2nd Edition, Garland Science, London, New York, 2011

[91] G. P. M. Leyson, W. A. Curtin, L. G. Hector, C. F. Woodward, Quantitative prediction of solute strengthening in aluminium alloys, Nature Materials 9 (9) (2010) 750-755. doi:10.1038/nmat 2813.

[92] G. Leyson, L. Hector, W. Curtin, Solute strengthening from first principles and application to aluminum alloys, Acta Materialia 60 (9) (2012) 3873 3884. doi:10.1016/j.actamat.2012.03.037.

[93] E. Rodary, D. Rodney, L. Proville, Y. Bréchet, G. Martin, Dislocation glide in model $\mathrm{Ni}$ ( $\mathrm{Al}$ ) solid solutions by molecular dynamics, Physical Review B 70 (5). doi:10.1103/PhysRevB. 70.054111.

[94] J. A. Yasi, L. G. Hector, D. R. Trinkle, Prediction of thermal cross-slip stress in magnesium alloys from direct first-principles data, Acta Materialia 59 (14) (2011) 5652-5660. doi:10.1016/j.actamat.2011.05. 040.

[95] L. Ventelon, F. Willaime, E. Clouet, D. Rodney, Ab initio investigation of the Peierls potential of screw dislocations in bcc Fe and W, Acta Materialia 61 (11) (2013) 3973-3985. doi:10.1016/j.actamat.2013.03. 012.

[96] A. Stukowski, V. V. Bulatov, A. Arsenlis, Automated identification and indexing of dislocations in crystal interfaces, Modelling and Simulation in Materials Science and Engineering 20 (8) (2012) 085007. doi: 10 . 1088/0965-0393/20/8/085007.

[97] N. Clement, P. Coulomb, Energies de défaut d'empilement et mécanismes de déformation dans les alliages nickel-chrome, Philosophical Magazine Series 830 (3) (1974) 663-672. doi:10.1080/ 14786439808206589 .

[98] D. E. Dominici, The Inverse of the Cumulative Standard Normal Probability Function, Integral Transforms and Special Functions 14 (4) (2003) 281-292. doi:10.1080/1065246031000081698. 